\title{
Functional genomics platform for pooled screening and mammalian genetic interaction maps
}

\author{
Martin Kampmann ${ }^{1,{ }^{*}}$, Michael C. Bassik ${ }^{1,2,{ }^{*} \text {, and Jonathan S. Weissman }}{ }^{1}$ \\ ${ }^{1}$ Department of Cellular and Molecular Pharmacology, California Institute for Quantitative \\ Biomedical Research, and Howard Hughes Medical Institute, University of California, San \\ Francisco, San Francisco, California, USA
}

\begin{abstract}
Systematic genetic interaction maps in microorganisms are powerful tools for identifying functional relationships between genes and defining the function of uncharacterized genes. We have recently implemented this strategy in mammalian cells as a two-stage approach. First, genes of interest are robustly identified in a pooled genome-wide screen using complex shRNA libraries. Second, phenotypes for all pairwise combinations of hit genes are measured in a double-shRNA screen and used to construct a genetic interaction map. Our protocol allows for rapid pooled screening under various conditions without a requirement for robotics, in contrast to arrayed approaches. Each stage of the protocol can be implemented in $~ 2$ weeks, with additional time for analysis and generation of reagents. We discuss considerations for screen design, and present complete experimental procedures as well as a full computational analysis suite for identification of hits in pooled screens and generation of genetic interaction maps. While the protocols outlined here were developed for our original shRNA-based approach, they can be applied more generally, including to CRISPR-based approaches.
\end{abstract}

\section{Keywords}

RNA interference; genetic interactions; epistasis; pooled screen; shRNA; mammalian cells

\section{INTRODUCTION}

The recent revolution in sequencing technology has facilitated the collection of massive amounts of human genomic sequence and expression data. To understand the function of the human genome and its role in disease, a complementary set of functional genomics tools is required. In particular, there is an acute need for methods that systematically discover pathway relationships between genes. One such method is the use of high-density genetic interaction maps, which report on pairwise measurements of the extent to which perturbing

\footnotetext{
Correspondence should be addressed to M.K. (martin.kampmann@ucsf.edu) and M.C.B. (bassik@stanford.edu).

${ }^{2}$ Current address: Department of Genetics, Stanford University, Stanford, CA, USA

*Equal contribution
}

\section{AUTHOR CONTRIBUTIONS}

M.K., M.C.B. and J.S.W. designed the study. M.K. and M.C.B. developed the experimental methods. M.K. developed the quantitative framework and scripts for computational analysis. M.K., M.C.B. and J.S.W. wrote the manuscript. 
one gene's function impacts the function of a second gene. These maps have proven to be powerful tools in haploid microorganisms such as yeast for understanding coordinated gene function and to identifying novel roles for poorly characterized genes ${ }^{1-8}$. Recently, we $\mathrm{e}^{9,10}$ and others ${ }^{11,12}$ have described efforts to construct and analyze genetic interaction maps in mammalian cells. Here, we describe a step-by-step protocol to conduct genetic screens and create genetic interaction maps based on our approach ${ }^{9,10,13}$.

Our current technology platform uses RNA interference (RNAi) to knock down genes. In the first stage, we define genes of interest in a pooled genome-wide screen, and in the second stage, we systematically determine all genetic interactions between these genes (Fig. 1). While RNAi proved a transformative technology for the study of mammalian cells, genome-wide RNAi screens have been plagued by false-positive and false-negative results ${ }^{14}$. To address these issues, we designed an ultra-complex genome-wide shRNA library in which each human gene is targeted by $\sim 25$ independent shRNAs. The library also contains thousands of matched negative controls. We have developed a quantitative framework for deriving shRNA phenotypes from pooled screens using such libraries, and for robustly detecting statistically significant hit genes, whose knockdown results in a phenotype of interest ${ }^{9}$. To determine genetic interactions between hits from the primary screen, shRNAs targeting the hit genes are individually cloned, batch-validated, and then used to construct a pooled double-shRNA library in a single ligation reaction. DoubleshRNA phenotypes are determined in a pooled screen and used to construct a genetic interaction map.

Two other approaches for large-scale genetic interaction maps in mammalian cells were recently published by other groups ${ }^{11,12}$. These alternative approaches are based on arrayed screening of combinations of siRNA or esiRNA reagents in separate wells. An advantage of arrayed approaches is the possibility to monitor complex phenotypes, such as cell morphology. However, specialized high-throughput equipment is required to implement them. Pooled screening strategies ${ }^{15-18}$, including ours ${ }^{9,10}$, have several important benefits, such as uniformity of growth conditions, and scalability, which is crucial to rapidly generate large genetic interaction maps and to compare genetic interaction maps across many different treatment conditions and genetic backgrounds. In addition, our library creation platform, quantitative framework and the computational pipeline for the analysis of pooled screens and the construction of genetic interaction maps ${ }^{9,10}$ applies more generally to other methodologies for interfering with gene expression, such as CRISPR-based modulation of gene transcription ${ }^{19-22}$.

\section{Applications of mammalian genetic interaction maps}

We have demonstrated that a mammalian genetic interaction map can be used to identify new protein complexes and identify novel roles for known complexes involved in the biology of the toxin ricin ${ }^{10}$. Other groups have shown that genetic interaction maps in mammalian cells can be used to gain insight into chromatin regulation ${ }^{12}$. We expect the potential uses of these maps to be very broad. The ability to query 100,000's of gene pairs in parallel for synthetic lethality in the context of cancer models promises to accelerate the identification of combination drug targets. The comparison of genetic interaction maps 
obtained under different conditions or in cells with different genetic backgrounds can reveal the rewiring of pathways in normal and disease states.

The protocol described here was used to produce our published genetic interaction map for factors controlling susceptibility of the human K562 leukemia cell line to ricin ${ }^{10}$. We are currently optimizing several aspects of our methodology for use in future studies. These aspects include the design of next-generation shRNA libraries, the use of bioreactors for large scale pooled screens, and the streamlined preparation of samples for deep sequencing, in which we are aiming to replace the gel purification step with size selection using solidphase reversible immobilization beads.

Limitations-Our strategy for primary screens has performed very well for screens comparing growth of untreated cells to growth of cells in the presence of a drug or toxin that causes an intermediate selective pressure. By contrast, treatments that kill more than 50\% of the cell population at a given time should be avoided, and instead, several rounds of moderate treatment should be used. In such screens, our method has successfully identified factors whose knock-down either sensitizes or protects cells from the treatment, compared to WT cells.

The single most important factor in obtaining reliable data from pooled screens is the representation of library elements in the cell population. While we aim for 1,000-fold to 4,000-fold representation, our pilot experiments have shown that higher representation results in even better signal-to-noise ratios ${ }^{10}$. A resulting limitation is that whole-genome screens using our methodology cannot easily be conducted where the scale of the pooled population is very limited - including screens such as mouse xenograft models.

Our strategy using ultra-complex libraries can robustly detect genes with even weak effects on the phenotype of interest ${ }^{10}$. However, it is possible that some relevant genes are not detected because the shRNA library does not contain enough shRNAs that knock down the gene sufficiently to observe the phenotype of interest. Furthermore, shRNAs that potently knock down genes essential for cell viability can get lost from the cell population before the beginning of the screen. This concern could be addressed by using inducible vectors (e.g. ref $^{23}$ ).

Finally, it is important to keep in mind that the typical selection protocol used in the primary screen (which often takes around 12-14 days) enables the detection of small differences in fitness or resistance to a selective pressure conferred by a given shRNA. This allows the identification of a wide range of genes of interest, which can then be characterized further in a systematic genetic interaction map. However, when validating hit genes individually, the observed effect in a short-term experiment (e.g. 2 days) may be comparatively small.

\section{Experimental Design}

\section{Pooled shRNA library}

It is assumed that the experimenter begins with a pooled high-coverage shRNA library in the lentiviral plasmid pMK1047 (ref. 10). Design and construction of such libraries has been 
described elsewhere ${ }^{9,10,13}$. For the purposes of screen design and analysis described here, essential features of these libraries are (1) 25 shRNAs per gene, and (2) large numbers ( 500-1000) of matched negative control shRNAs per sublibrary designed using the same rules as for the targeted shRNAs. The plasmids we are currently using for primary screens, batch retests of shRNAs and double-shRNA screens are annotated in Fig. 2. The vectors encode mCherry and puromycin, which allow monitoring of viral infection and selection for the presence of stably integrated virus.

\section{Considerations for screen design}

The protocol presented here applies to a screen based on growing cells under standard conditions or in the presence of selective pressure, such as addition of a drug or toxin ${ }^{10}$. Quantification of the frequencies of cells expressing a specific shRNA in starting populations, untreated and treated populations can be used to determine different shRNA phenotypes, which we have previously defined quantitatively ${ }^{9}$. Briefly, gamma phenotypes quantify the effect of an shRNA on cell growth in the absence of treatment, tau phenotypes quantify the effect of an shRNA on cell growth in the presence of treatment, and rho phenotypes quantify the effect of an shRNA on resistance to the treatment. While growth and selection conditions must clearly be optimized for each screen and cell type, several general principles should guide screen design:

- Selection should be set up so that a sufficient dynamic range between treated and untreated conditions can be expected. In our screens, a difference of around 6 net doublings between treated and untreated conditions worked well to see significant differences. With such a protocol, an shRNA conferring complete resistance to the selective pressure should be enriched $2^{6}=64$-fold relative to a negative control shRNA.

- Given the complexity of the shRNA library, it is extremely important to avoid tight population bottlenecks at each stage of the experiment: lentiviral infection, screen selection, and preparation of the sequencing sample. We aim to maintain 1,000-fold representation of each library shRNA on average at each stage. For example, for a screen of an shRNA library with 50,000 different shRNAs, the cell population should be maintained at 50 million cells or more. In particular, when designing a screen, care should be taken to avoid selective pressures that are so high that a large fraction of the cell population is killed. We aim for selective pressures that do not kill more than $50 \%$ of the population at any time point during the screen.

- Considerations for library representation also affect the numbers of double-shRNAs that can be queried for the construction of a genetic interaction map. For example, if the screen is carried out in a volume of 21 at a cell concentration of 500,000 cells $/ \mathrm{ml}$, i.e. a population of 1 billion cells, a representation of 1,000-fold would correspond to a complexity 1 million, or all pairwise combinations of 1,000 different shRNAs. To get better quantitative data, we typically choose a representation of 4,000-fold for double-shRNA screens, which would reduce the number of shRNAs in the above example to $500 \times 500$. We typically include 12 independent negative control shRNAs, which then form combinations with other negative control shRNAs (used to derive the WT phenotype in the screen) and 
combinations with gene-targeting shRNAs (used to calculate single-gene phenotypes).

- Suspension cell lines facilitate culturing of large cell numbers, since they can easily be grown in large spinner flasks or bioreactors, and do not require trypsinization for passaging, but can simply be diluted. We had good experiences with the human K562 leukemia cell line, which does not grow in clusters and grows stably over many generations. Other suspension lines, including B-cell, T-cell and myeloma lines have also worked well in our hands. Adherent cell lines can be seeded on microcarriers and then grown in spinner flasks or bioreactors.

- We have successfully detected both positive and negative selection. It can however be more challenging to confidently detect shRNAs that cause cells to drop out of the screen, especially if they also affect growth under standard conditions, since phenotypes for such shRNAs are then calculated based on low numbers of reads in the sequencing data, which inherently will increase statistical noise.

\section{Considerations for sample preparation}

Following the screen, genomic DNA is isolated from the different cell populations and the shRNA-encoding cassettes are PCR-amplified to generate a sample for deep sequencing. Our protocol was designed with the following goals:

- Adequate representation of the complex cell population should be maintained during library preparation. Genomic DNA is isolated from a number of cells that is 1,000-fold the number of different shRNAs in the screened library. To reduce the amount of genomic DNA used as a PCR template, we size-fractionate the DNA by a restriction enzyme digest (with PvuII) followed by gel purification of the size range around the expected fragment size of the shRNA-expression cassette. We are currently testing an alternative approach based on DNA size selection with solidphase reversible immobilization beads, which may be implemented in future versions of this protocol.

- During sample preparation from the primary screen, the DNA region encoding the shRNA is PCR-amplified. We found that amplification of this region creates bias against GC-rich shRNAs - presumably because the DNA is more likely to form secondary structures that interfere with amplification. Therefore, it is important that the exact same PCR protocol is used to amplify samples that will be compared during data analysis. Future versions of our library will implement a restriction site in the shRNA loop-encoding region to enable linker ligation followed by half-mer amplification, thus circumventing amplification of the full shRNA-encoding region. Other groups have previously developed analogous strategies ${ }^{16,24}$.

- In the PCR-amplified material, the shRNA-encoding region is highly enriched compared to its abundance in pre-PCR genomic DNA. Therefore it is critical to avoid cross-contamination of pre-PCR material with any post-PCR material. Ideally, the pre-PCR steps should be performed using separate equipment (e.g., pipettes, vacuum manifolds) from that used for post-PCR steps. 
Controls

- For pilot experiments, it is helpful to use positive and negative control shRNAs to assess the dynamic range of screening conditions. Cells infected with control lentivirus expressing mCherry can be mixed with WT cells, and used to track changes in populations under mock selection conditions ${ }^{10}$.

- To distinguish the effect shRNA have on cell growth under standard (untreated) conditions from treatment-specific effects, experiments should be designed to compare the population at the time point before selection ( $\mathrm{t} 0$ ), the treated population at the end point of the experiment, and a control untreated population that grew for the same time in parallel.

- After infection of cells with the lentiviral library, vials of cells representing the library diversity at $\sim 1000$-fold should be frozen to be used as t0 controls, backup vials, and replicate experiments.

\section{MATERIALS}

\section{REAGENTS}

- Genome-wide shRNA library (e.g. in plasmid pMK1047, as described in refs. 9,10. Plasmid pMK1047 will be provided on request.)

- Target cell line in which the screen is conducted

- $\quad$ 293T cells (ATCC cat \# CRL-3216)

- Heat-inactivated FBS (Hyclone Characterized, Thermo Scientific, Cat\# SH30071.03)

- DMEM-High glucose (Sigma, cat\# D5796)

- $\quad$ RPMI w/HEPES modification (Sigma, cat\# R5886)

- 100x Penicillin/Streptomycin (Sigma, cat\# P4333)

- 100x L-glutamine (Sigma, cat\# G7513)

- $\quad$ PBS (Sigma, cat\# D8537)

- Trypsin (Sigma, cat\#T3924)

- Puromycin (Sigma, cat\#P8833-10MG)

- $\quad$ Mirus LT1 Transfection agent (Mirus, cat \# MIR 2300)

- QIAamp DNA Blood Maxi Kit (Qiagen, cat \# 51192)

- Qiagen miniprep kit (Qiagen, cat \# 27106)

- $\quad$ Qiagen Buffer PB (Qiagen, cat \# 19066)

- Qiagen Gel extraction kit (Cat No. 28704 or 28706)

- Qiagen Minelute kit (Cat \# 28204)

- Qiagen QIAquick PCR purification kit (Cat No. 28104 or 28106) 
- $\quad$ Ethidium bromide (Biorad, cat \# 161-0433)

- $\quad$ BstXI (NEB, cat \# R0113S)

- $\quad$ PvuII (NEB, cat \# R0151S)

- High-sensitivity DNA kit (Agilent Technologies, cat. no. 5067-4626)

- $\quad$ TruSeq SBS v3 kit, 50 cycles (Illumina, cat. no. FC-401-3002)

- $\quad$ TruSeq SR Cluster Kit v3, cBot, HS (Illumina, cat. no. GD-401-3001)

- Phusion ${ }^{\circledR}$ High-Fidelity PCR Kit with 5X HF buffer (NEB Cat No. E0553L)

- $\quad$ dNTPs (e.g. NEB N0447L)

- $\quad$ Polybrene (Sigma cat \# H9268-5G)

- DMSO (Sigma Hybrimax, cat\# D2650-5X5ML)

- Mr. Frosty Freezing container (Fisher, cat\# 15-350-50)

- $\quad$ Tris base (Fisher, cat \# BP152-5)

- $\quad$ Boric acid (Sigma, cat \# B6768)

- $\quad$ Acetic acid (Fisher, cat \# A38-212)

- $\quad$ EDTA (Sigma, cat \# 03660)

- $\quad$ Bromophenol blue (Bio-rad, cat \# 161-0611)

- Xylene cyanol (Sigma, cat \# X4126)

- $\quad$ Sucrose (Sigma, cat \# 84097)

- HEPES (Sigma, cat \# H3375)

- Magnesium acetate (Sigma, cat \# M0631)

- $\quad$ Potassium acetate (Sigma, cat \# P5708)

- $\quad$ Agarose (Research Products International, Cat \# A20090-500)

- 20\% Polyacrylamide/TBE gels, 1.0mm, 12-well (Invitrogen, cat \# EC63152BOX)

- $\quad 1 \mathrm{~kb}+$ DNA ladder (Invitrogen, cat \# 10787-018)

- D-tube midi electroelution columns (EMD cat \# 71507-3)

- $\quad$ Milli-Q purified $\mathrm{H}_{2} \mathrm{O}$

- Oligonucleotides from IDT Technologies (see sequences in Table 1)

- MegaX DH10 T1R Electrocompetent Cells (Invitrogen, cat \# C6400-03)

- Lentiviral packaging plasmids pRSV, pMDL, pVSVG (obtained from the Jacks lab, described in refs ${ }^{25,26}$ )

- Plasmid pMK1200 (ref. 9), which we will provide on request.

- $\quad$ T4 DNA Ligase + buffer (NEB, cat \# M0202T) 
- DH5a cells, subcloning efficiency (Life Technologies, cat\#18265-017)

- Carbenicillin (Research Products International, cat \# C46000)

- $\quad$ agar (Fisher cat \# DF0145070)

- bacto tryptone (Fisher cat \# DF0123-07-5)

- $\quad$ sodium chloride (Fisher cat \# S271-10)

- yeast extract (Fisher cat \# DF0127071)

- $\quad$ SOC (Life Technologies, cat\#15544-034)

- $\quad$ Pasteur pipets (glass) (VWR cat \# 14672-380)

- Qiagen Gigaprep Kit (Qiagen, cat\#12191)

- Qtray plates (Molecular devices, cat \# X6023)

- 1-mm gap cuvette for electroporation (USA Scientific, cat \# 9104-1050)

\section{EQUIPMENT}

- $\quad$ 15-cm Tissue culture plates (Fisher cat \# 12-565-100)

- $\quad$ T150 flasks (Fisher, cat\# 1012634)

- 6-well plates (Corning, cat\# 3506)

- $30 \mathrm{~mL}$ syringe (BD, Fisher cat \# 14-829-48)

- $\quad 0.45$ um syringe filter with luer lock (Millipore, SLHV033RB)

- 96-well PCR plates (Bio-Rad, cat \# MLP-9601)

- 15 and $50 \mathrm{~mL}$ falcon conical tubes (Fisher, cat \# 14-959-53A and 14-432-22)

- Owl A1 Large Gel System (Thermo Scientific, cat \# A1)

- Gel combs creating large volume wells (e.g. $2 \mathrm{ml}$ ). These can be created by taping together standard gel combs, or, if a 3D printer is available, by constructing a customized comb. We will provide a file for this on request.

- Invitrogen Xcell SureLock® Mini-Cell (Invitrogen, cat \# EI0001)

- Tissue culture benchtop centrifuge with plate buckets

- Qiagen vacuum manifold (Qiagen cat \# 19413)

- Illumina HiSeq next generation sequencer (Illumina)

- Thermal cycler (Bio-Rad, cat. no. 170-9713)

- 2100 BioAnalyzer (Agilent Technologies, cat. no. G2940CA)

- Computer. The scripts described here have been developed for use on a computer with a MacOS operating system. Use on computers with other operating systems may require adaptation of the scripts.

- $\quad$ Razors (VWR, cat. no. 55411-050) 
- Low-power UV gel box

- $\quad$ Electroporator (e.g. Bio-rad cat \# 165-2662)

- $\quad$ Bunsen burner (e.g. Fisher cat \# S49107)

\section{REAGENT SETUP}

293T growth medium-To 450mL DMEM-High glucose, add 50mL heat-inactivated FBS (10\% (vol/vol) final), $5 \mathrm{~mL}$ Penicillin/Streptomycin (100x), and $5 \mathrm{~mL}$ Glutamine (100x). Store at $4 \mathrm{C}$ for up to 1 month.

$4 \mathrm{mg} / \mathrm{mL}$ polybrene-Add $40 \mathrm{mg}$ polybrene to $10 \mathrm{~mL}$ Millipore water, filter through a 0.2 um filter, and freeze in aliquots at $-80 \mathrm{C}$, and store indefinitely.

Freezing medium-Prepare freezing medium by adding $5 \mathrm{~mL}$ DMSO to heat-inactivated FBS (10\% DMSO final), and filter through a 0.2 um filter. Store at $-20 \mathrm{C}$ for up to 1 year.

1X TAE buffer-Prepare 1L 1X TAE by combining 4.84g Tris-base, $1.14 \mathrm{~mL}$ acetic acid, and $2 \mathrm{~mL} 0.5 \mathrm{M}$ EDTA $\mathrm{pH} 8.0$; adjust to $1 \mathrm{~L}$ with milli-Q water [40mM Tris, $20 \mathrm{mM}$ acetic acid, 1mM EDTA, pH 8.0 final]. Store at room temp 6 months.

10x DNA loading buffer-Prepare $10 \mathrm{~mL}$ 10x DNA loading buffer by combining $6.5 \mathrm{~g}$ sucrose, $100 \mathrm{uL} \mathrm{1M}$, Tris pH 7.5, $200 \mathrm{uL}$ 0.5 M EDTA pH8.0, $2 \mathrm{mg}$ bromophenol blue, and $2 \mathrm{mg}$ xylene cyanol; adjust to $10 \mathrm{~mL}$ with milli-Q water [0.65 g/ml sucrose, $10 \mathrm{mM}$ Tris $\mathrm{pH}$ 7.5, $10 \mathrm{mM}$ EDTA, $0.02 \%$ (w/v) bromophenol blue, $0.02 \%$ (w/v) xylene cyanol final].

1X TBE buffer-Prepare 1L 1X TBE by combining 10.8g Tris, $5.5 \mathrm{~g}$ Boric acid, $4 \mathrm{~mL}$ $0.5 \mathrm{M}$ EDTA ( $\mathrm{pH} 8.0$ ), and adjust volume to $1 \mathrm{~L}$ with milli-Q water. [89 mM Tris, $89 \mathrm{mM}$ boric acid, 2 mM EDTA, pH 8.0 final] Store at room temp for 6 months.

2X Annealing buffer-Prepare 100mL 2X Annealing buffer by combining $1.96 \mathrm{~g}$ potassium acetate, $12 \mathrm{~mL} 0.5 \mathrm{M}$ HEPES-KOH pH 7.4, and $0.86 \mathrm{~g}$ magnesium acetate, and adjust to $100 \mathrm{~mL}$ with milli-Q water. [200 $\mathrm{mM}$ potassium acetate, $60 \mathrm{mM}$ HEPES-KOH pH 7.4, $4 \mathrm{mM}$ magnesium acetate final]. Store at room temp for 6 months.

Qtray Agar plates-Prepare LB agar: $15 \mathrm{~g} / \mathrm{l}$ agar, $10 \mathrm{~g} / \mathrm{l}$ bacto trypton, $5 \mathrm{~g} / \mathrm{l}$ sodium chloride, $5 \mathrm{~g} / \mathrm{l}$ yeast extract, autoclave, cool down while stirring. When the temperature is below $50{ }^{\circ} \mathrm{C}$, add $75 \mathrm{ug} / \mathrm{ml}$ carbenicillin. Pour into Qtray plates, $200 \mathrm{ml}$ per plate.

LB Agar plates-Prepare LB agar: $15 \mathrm{~g} / \mathrm{l}$ agar, $10 \mathrm{~g} / \mathrm{l}$ bacto trypton, $5 \mathrm{~g} / \mathrm{l}$ sodium chloride, $5 \mathrm{~g} / \mathrm{l}$ yeast extract, autoclave, cool down while stirring. When the temperature is below 50 ${ }^{\circ} \mathrm{C}$, add $100 \mathrm{ug} / \mathrm{ml}$ carbenicillin. Pour into $10 \mathrm{~cm}$ round plates.

Glass spreading rod-In the flame of a Bunsen burner, heat the region of a glass Pasteur pipet about $5 \mathrm{~cm}$ below the tip. After a few seconds, the pipet will bend in the heated region, remove it from the flame. Now heat the very tip of the pipet to bend it towards the thick 
stem of the pipet. The resulting glass spreading rod allows scraping of colonies from agar plates without scratching the agar surface.

\section{EQUIPMENT SETUP}

\section{Software and file installation}

- Install bowtie 27 , which can be downloaded for free at http://sourceforge.net/ projects/bowtie-bio/files/bowtie/1.0.0/.

- Install python (www.python.org), or, in order to use interactive graphics, install the Canopy Python package from Enthought, Inc., which is free for academic use (https://www.enthought.com/products/canopy/academic/).

- To generate tab-delimited text files, we recommend the use of a command-line text editor, like vim (http://www.vim.org).

- Download an archive containing the custom scripts used in this protocol, as well as example data, from gimap.ucsf.edu. Unzipping of the archive will create the subdirectory structure referred to in this protocol.

- Download Homo_sapiens.gene_info.gz from ftp://ftp.ncbi.nlm.nih.gov/gene/ DATA/GENE_INFO/Mammalia/, unzip the file and move it to subdirectory LUT.

- Download and install the Pycluster module for python from http://bonsai.hgc.jp/ $\sim$ mdehoon/software/cluster/software.htm\#pycluster

- Download and install Cluster 3.0 (ref. ${ }^{28}$ ) from http://bonsai.hgc.jp/ mdehoon/ software/cluster/software.htm.

- Download Java Tree View ${ }^{29}$ from http://sourceforge.net/projects/jtreeview/files/.

- Create a fasta file for the shRNA library you used for the primary screen as follows: Each entry describes one shRNA. Each shRNA name should begin with the Entrez Gene ID, followed by a double underscore, and a number or other information that uniquely identifies the shRNA, e.g. 9064_NM_004672.3_24. Names of negative control shRNAs should have a 0 instead of an Entrez Gene ID, e.g. $0 \_0 \_1$. The nucleotide sequence included for each shRNA is the reversecomplement of the shRNA guide strand for the shRNA in your shRNA library. (The length of each sequence should be $22 \mathrm{nt}$, so in case your guide strand is shorter, include the reverse-complement sequence of the constant nucleotides following the guide strand in your shRNA construct.) To create a bowtie index from this file, use the bowtie-build command. For example, if the path to bowtiebuild on your computer is/Applications/bowtie-0.12.3/bowtie-build, and the name of the fasta file for your shRNA library is LibraryName.fa, enter the following on the command line:

/Applications/bowtie-0.12.3/bowtie-build LibraryName. fa LibraryName 
Six output files are created, move them to the subdirectory indices/. In the script align_primary.py (subdirectory scripts), specify the path to bowtie on your computer in line 4.

\section{PROCEDURE}

\section{Lentivirus preparation and infection - TIMING 7-10 d}

! CAUTION All lentivirus work should be performed in BSL2 containment; wear appropriate protective clothing, and dispose of lentivirus-infected materials using bleach.

1 Trypsinize healthy, logarithmically dividing $293 \mathrm{~T}$ cells and plate $7.5 \times 10^{6}$ in a $15-\mathrm{cm}$ plate in $30 \mathrm{ml}$ of $293 \mathrm{~T}$ growth medium. Steps $1-5$ describe the handling of one $15 \mathrm{~cm}$ plate, which is on appropriate scale for an shRNA library with a complexity of up to $\sim 20,000$. Scale up the number of plates for larger shRNA libraries as necessary.

2 Transfection: $24 \mathrm{~h}$ after plating the cells, prepare transfection mix by add $48 \mu \mathrm{l}$ Mirus LT1 to $1300 \mu$ DMEM (no additives), mix and wait $5 \mathrm{~min}$. Add a mixture of $8 \mu \mathrm{g}$ pooled shRNA library (e.g. in plasmid backbone pMK1047) and $8 \mu \mathrm{g}$ of an equal mix of packaging plasmids (pMDL, pRSV, pVSVG). Mix, wait 20 min, and then add dropwise to the $15 \mathrm{~cm}$ plate.

3 $\quad 72 \mathrm{~h}$ after transfection, harvest virus by aspiration into a $30 \mathrm{ml}$ luer-lock syringe (no needle), and then filtration through $0.45 \mu \mathrm{m}$ filter into $50 \mathrm{ml}$ conical tube. We prepare virus fresh for each infection, although in principle virus can be concentrated, titered, and stored at $-80^{\circ} \mathrm{C}$.

\section{PAUSE POINT}

4 | Transduction: for suspension cells, infect cells with the virus library by resuspending $35 \times 10^{6}$ cells in $60 \mathrm{ml}$ lentiviral supernatant supplemented with $8 \mathrm{ug} / \mathrm{ml}$ polybrene, and distributing this mixture into the wells of a 6-well plate. Centrifuge at $1000 \mathrm{xg}$ for $2 \mathrm{~h}$ at $33^{\circ} \mathrm{C}$. If using adherent cells, , split cells the day before infection, and infect cells by simple addition of virus supernatant to the growth media; no centrifugation or polybrene is necessary (skip step 5). The exact parameters may need to be adjusted based on the target cell line.

CRITICAL STEP Optimize infection for each cell type by adjusting the amount of virus added to the cells. The initial multiplicity of infection (monitored as the fraction of mCherry-expressing cells by microscopy or flow cytometry) should be $\sim 30-40 \%$ to minimize double-infection of cells.

5 Carefully pipet the suspension cells in the 6-well plate up and down with a $5 \mathrm{ml}$ serological pipet. Transfer all cells to a $50 \mathrm{ml}$ conical tube and spin them at $400 \mathrm{~g}$ for $5 \mathrm{~min}$ at room temp. Remove viral supernatant by aspiration and resuspend cells in fresh growth medium. Transduction during spin step is sufficient for infection.

6| Allow the cells 2 days of recovery before selecting them for stable integration of virus with puromycin. 
CRITICAL STEP Titer the dose of puromycin for each cell line to identify the minimum necessary dose to kill $\sim 80-90 \%$ of cells to avoid selection for superinfected cells.

7| The selection of cells with stably integrated virus can can be monitored as an increase in the fraction of mCherry-expressing cells by flow cytometry or microscopy. Once $\sim 80 \%$ of cells are mCherry-positive (typically after 1-3 days of selection), cells should be removed from puromycin and expanded in fresh growth medium. Vials of cells should be frozen to be used as t0 controls, backup vials, and for replicate experiments. The number of cells in each aliquot should be chosen such that the average representation of shRNAs in each library is 1,000-fold. (E.g., for a library with 50,000 shRNAs, 50 million infected cells should be frozen in each aliquot.)

PAUSE POINT After expansion and freezing vials, cells can be stored indefinitely in liquid nitrogen before screening.

\section{? TROUBLESHOOTING}

\section{Conducting a screen - TIMING (varies from 1-21 d depending on selection strategy)}

8| Cells are subjected to selective pressure (duration, dose, strategy are dependent on individual experiment); see experimental design section for considerations.

9| For a screen based on growth or survival under a selective pressure, keep track of the number of cell doublings for the treated and untreated (control) cell populations. The fraction of mCherry-positive cells may change during the screen, as monitored by flow cytometry. In that case, calculate the number of doublings for the uninfected (mCherry-negative) cells, since these represent the wild type.

10 At the end of the screen, freeze down multiple vials of cells for selected and unselected conditions. As for t0 aliquots, the number of cells in each aliquot should be chosen such that the average representation of shRNAs in each library is 1,000fold. (E.g., for a library with 50,000 shRNAs, 50 million infected cells should be frozen in each aliquot.)

\section{Preparing samples for deep sequencing - TIMING $3 \mathrm{~d}$}

11 Genomic DNA is prepared using Qiagen DNA Blood Maxi kits. We chose this method for ease of use and reproducibility, although alternative methods for DNA purification could also be used. 1 column is used to extract DNA from 100 $\times 10^{6}$ cells, and number of columns is scaled accordingly.

12| Preheat a water bath to $70^{\circ} \mathrm{C}$.

13 Thaw cell aliquots by incubating them in a $37^{\circ} \mathrm{C}$ water bath.

14 | Resuspend cells in $25 \mathrm{ml}$ PBS into a $50 \mathrm{ml}-$ Falcon tube and pellet by centrifugation $5 \mathrm{~min}$ at $400 \mathrm{~g}$. 
15 Based on the number of total cells per sample, extract genomic DNA using the QIAamp DNA Blood Maxi kit according to the manufacturer's instructions, with the specifications described in the table below. If more than $300 \times 10^{6}$ cells are present in the sample, split the cells into $>1$ Falcon tube before starting. To the manufacturer's instructions apply the following minor modifications: repeat step 6 (leave first eluate in tube) and pipet up and down to ensure genomic DNA is in solution; in step 9 , spin 2 minutes at $3800 \times \mathrm{g}$; in step 10 , spin 20 minutes at $3800 \times \mathrm{g}$; in the elution step add $0.8 \mathrm{ml} \mathrm{EB}$ per column, incubate for $5 \mathrm{~min}$, and centrifuge for $5 \mathrm{~min}$ at $3800 \times \mathrm{g}$.

\begin{tabular}{l|l|l|l}
\hline Number of cells: & $<\mathbf{1 0 0} \times \mathbf{1 0}^{\mathbf{6}}$ & $<\mathbf{2 0 0} \times \mathbf{1 0}^{\mathbf{6}}$ & $<\mathbf{3 0 0 \times \mathbf { 1 0 } ^ { \mathbf { 6 } }}$ \\
\hline Resuspend in PBS & $5 \mathrm{ml}$ & $10 \mathrm{ml}$ & $15 \mathrm{ml}$ \\
\hline Add Qiagen protease & $500 \mathrm{ul}$ & $1,000 \mathrm{ul}$ & $1,500 \mathrm{ul}$ \\
\hline Add buffer AL & $6 \mathrm{ml}$ & $12 \mathrm{ml}$ & $18 \mathrm{ml}$ \\
\hline Add ethanol & $5 \mathrm{ml}$ & $10 \mathrm{ml}$ & $15 \mathrm{ml}$ \\
\hline Split to how many columns: & 1 & 2 & 3 \\
\hline
\end{tabular}

16 Measure genomic DNA concentration (typically $\sim 1 \mathrm{mg} / 100 \times 10^{6}$ cells or more).

PAUSE POINT Eluted genomic DNA can be frozen at $-20^{\circ} \mathrm{C}$ indefinitely.

17 Set up a restriction digest of the genomic DNA in a 15-ml Falcon tube. For each $1 \mathrm{mg}$ genomic DNA, adjust volume with Qiagen buffer EB to 1,500 $\mu$, mix with $169 \mu \mathrm{l} \mathrm{NEB}$ Buffer 2 and add $20 \mu \mathrm{l}$ of PvuII. Incubate overnight at $37^{\circ} \mathrm{C}$.

18 Pour $400 \mathrm{ml} 0.8 \%$ agarose gel in TAE containing $32 \mu \mathrm{l} 10 \mathrm{mg} / \mathrm{mL}$ ethidium bromide in an Owl A1 large gel system using gel combs creating large wells.

!Caution Ethidium Bromide is mutagenic, and should be handled with care and disposed of properly

19| After gel has solidified, slowly and carefully remove gel comb. Add TAE to the gel chamber so that buffer surface is $\sim 1-2 \mathrm{~mm}$ below the top surface of gel.

CRITICAL STEP $1.5 \mathrm{ml}$ volume wells generated with custom gel combs are very fragile. Tearing of the wells can cause sample leak and cross-contamination of samples.

CRITICAL STEP Large sample volumes cannot be neatly loaded if gel is under buffer, so it is necessary to maintain the gel surface exposed.

Carefully add sample in loading buffer to well, and run alongside $2.5 \mu \mathrm{g} 1 \mathrm{~kb}+$ DNA ladder (in an adjacent small well) at $220 \mathrm{~V}$ for $\sim 80 \mathrm{~min}$.

20|Visualize PCR products on a low-power UV gel box. Cut a wide gel slice spanning between the $1 \mathrm{~kb}$ and 1.6kb markers (Fig. 3a) and transfer to a $50-\mathrm{mL}$ tube. Gel slices are typically $\sim 12-\mathrm{mL}$ in volume. 
21 Add 3 gel volumes of buffer QG to the gel slice and incubate in a water bath at $55^{\circ} \mathrm{C}$ with occasional agitation until the gel is fully dissolved.

22 Add 1/100 of the total buffer QG + gel volume of 3M sodium acetate $\mathrm{pH} 5.2$ and mix.

23 Purify genomic DNA by collecting the DNA/QG mixture onto Qiagen gel extraction columns ( 4 columns per $100 \times 10^{6}$ cells starting material), using a Qiagen vacuum manifold.

CRITICAL STEP Separate vacuum manifolds should be used for pre-PCR and post-PCR DNA purification to avoid cross-contamination with trace amounts of DNA from other experiments. Great care should be taken to thoroughly wash the manifold with water after each use.

24 Wash columns twice with $800 \mu$ Qiagen buffer PE, then remove from vacuum manifold and spin $1 \mathrm{~min}$ at $14,000 \mathrm{rpm}$ in an eppendorf centrifuge to dry membranes.

25 Elute each column with $50 \mu \mathrm{EB}$.

26 Measure concentration of post-digest DNA (typically $\sim 15-20 \mu \mathrm{g}$ per $50 \times 10^{6}$ cells).

PAUSE POINT Eluted DNA can be frozen at $-20^{\circ} \mathrm{C}$ indefinitely.

27 PCR amplify the shRNA-encoding cassette, introducing Illumina adapter sequences with different indexes. For each sample that will be sequenced in the same lane, choose a unique forward primer index from the following set described in Table 1: oMK312, oMK313, oMK314, oMK315, oMK316, oMK317, oMK318, or oMK319. Indices should be chosen so that unique barcode sequences (see Table 1) can be distinguished when samples are pooled for Illumina sequencing. For all samples, use the same reverse primer, oMCB800 (Table 1). A typical PCR reaction total volume for DNA from $50 \times$ $10^{6}$ cells is between 1 and $2 \mathrm{ml}$, i.e. between 10 and 20 times the $100 \mu \mathrm{lPCR}$ reaction described below. Assemble the following PCR reaction, scaling the total volume to amplify the full amount of genomic DNA from Step 26:

\begin{tabular}{lcc}
\hline Compound & amount per sample & final concentration \\
\hline Size-fractionated DNA & $1.5 \mu \mathrm{g}$ & $150 \mathrm{ng} / \mathrm{ul}$ \\
Forward primer $(100 \mu \mathrm{M}$ stock $)$ & $0.8 \mathrm{ul}$ & $0.8 \mathrm{uM}$ \\
Reverse primer oMCB800 $(100 \mu \mathrm{M}$ stock $)$ & $0.8 \mathrm{ul}$ & $0.8 \mathrm{uM}$ \\
DMSO & $3 \mathrm{ul}$ & $3 \%$ \\
dNTPs (10 mM stock) & $2 \mathrm{ul}$ & $200 \mathrm{uM}$ \\
$\%$ X HF buffer & $20 \mathrm{ul}$ & $1 \mathrm{X}$ \\
Phusion polymerase & $1 \mathrm{ul}$ & \\
Water & up to $100 \mathrm{ul}$ & N/A \\
\hline
\end{tabular}


CRITICAL STEP Maximum of $1.5 \mu \mathrm{g}$ genomic DNA can be amplified in 100 $\mu$ l.

28 Distribute the PCR mix into a 96-well PCR plate, $100 \mu$ l per well, and perform PCR with following conditions:

\begin{tabular}{|l|l|l|l|l|}
\hline Cycle number & Denature & Anneal & Extend & Hold \\
\hline 1 & $98{ }^{\circ} \mathrm{C}, 2 \mathrm{~min}$ & & & \\
\hline $2-24$ & $98{ }^{\circ} \mathrm{C}, 30 \mathrm{~s}$ & $56^{\circ} \mathrm{C}, 15 \mathrm{~s}$ & $72^{\circ} \mathrm{C}, 15 \mathrm{~s}$ & \\
\hline 25 & & & $72^{\circ} \mathrm{C}, 10 \mathrm{~min}$ & \\
\hline 26 & & & & $4{ }^{\circ} \mathrm{C}$ \\
\hline
\end{tabular}

29| Collect the PCR reactions from the 96-well plate into a $15 \mathrm{ml}$ conical tube. Once the material has been mixed, only a fraction (typically $800 \mathrm{ul}$ ) needs to be processed, the rest can be stored as a backup sample. Mix 1 part PCR reaction with 4 parts Qiagen buffer PB, and add 1/100 volume of 3M Sodium acetate $\mathrm{pH}$ 5.2 .

30 | Purify the sample/PB mix over a Qiagen PCR purification column, following the manufacturer's instruction, and elute in 50 ul buffer EB.

31 Load the PCR product in DNA loading buffer onto a $20 \%$ polyacrylamide/TBE gel, distributing $20 \mathrm{uL}$ into adjacent wells of a 12-well gel, and run for $1 \mathrm{~h}$ at $180 \mathrm{~V}$ in $0.5 \mathrm{X}$ TBE. Once the sample has been mixed, only a fraction of the sample (typically 20\%) needs to be loaded onto the gel.

32 Remove gel from casing and stain in 50ml $0.5 X$ TBE with $5 \mathrm{ul}$ of $10 \mathrm{mg} / \mathrm{ml}$ ethidium bromide for $5 \mathrm{~min}$ on a rocking platform.

33 Visualize PCR products on a low-power UV gel box (Fig. 3b). The correct products should run at $\sim 250 \mathrm{bp}$. Excise this band using a razor blade.

\section{? TROUBLESHOOTING}

34 Combine all slices from a single sample into a D-tube midi electroelution column (pre-soaked in $800 \mu \mathrm{l} 0.5 \mathrm{xTBE}$ for $5 \mathrm{~min}$ and emptied), add $800 \mu \mathrm{l}$ fresh $0.5 \mathrm{X}$ TBE and close.

CRITICAL STEP Make sure the lid is closed finger-tight.

35 Electroelute in TBE for $1.5 \mathrm{~h}$ at $160 \mathrm{~V}$; reverse electrode polarity for the last 2 minutes of electroelution to ensure DNA does not stick to membrane.

CRITICAL STEP Make sure the tubes stay submerged in TBE throughout the electroelution time.

36 Collect electroeluted DNA, mix 1 part PCR product with 4 parts Qiagen buffer $\mathrm{PB}$, and add 1/100 volume of 3M sodium acetate $\mathrm{pH}$ 5.2. 
37 | Purify PCR product on 1 Qiagen gel extraction column using a vacuum manifold, wash column with $1 \mathrm{ml}$ Qiagen buffer PE, and elute in $100 \mathrm{ul}$ buffer EB.

38 Verify the size and concentration of the purified PCR product using procedures recommended by the sequencing facility (such as qPCR or bioanalyzer), and sequence on an Illumina HiSeq using primer oMK132. This primer should be added to the primer mix provided by Illumina as follows: for each lane, $240 \mathrm{ul}$ of sequencing mix HP1 (provided with Illumina sequencing reagents) should be mixed with $1.2 \mu \mathrm{l}$ of $100 \mu \mathrm{M}$ oMK132. A 1000 -fold representation of library shRNAs by sequencing reads is recommended, e.g. for a library with 50,000 different shRNAs, 50 million reads should be obtained.

\section{Data analysis for the primary screen - TIMING 1-4d}

39 In the subdirectory growth, create a tab-delimited text file containing, on the first line, the number of doublings untreated WT cells completed during the experiment (Step 9) ('G'), and, separated by a tab, the difference between that number and the number of doublings treated WT cells completed during the experiment (' $K$ '). As an example, we have provided the file Example_Primary_GK.txt.

40 Move the sequencing results files, in fasta or Illumina fastq format, to the subdirectory sequencing.

41 Open a Terminal window and on the command line, change to the directory scripts, and enter the IPython console with the following command:

ipython --pylab

The Enthought python distribution enables interactive graphical data display. If you do not want to use this feature, you can execute all scripts directly from the terminal command line by replacing "run" with "python" in the following command line examples (Steps 42-46). In that case, where graphical output is created as part of the program pipeline, the pop-up window with the graph needs to be closed for the program to proceed.

42 $\mid$ Run the script crop_reads.py to process the sequencing data with the following command-line arguments:

- Name of the sequencing data file, e.g. ../sequencing/Sample.fastq

- Name of the output file, e.g. ../cropped/Sample.fa

- Number of bases after which the sequencing read should be truncated. For the analysis of primary screen samples, 22 is sufficient.

Example command line:

In [1]: run crop_reads.py ../sequencing/Sample.fastq ../cropped/Sample.fa 22 
43 $\mid$ Run the script align_primary.py to align the cropped reads to the shRNA library reference with the following command-line arguments:

- Cropped sequencing data file, e.g. ../cropped/Sample.fa

- Prefix for the output files generated by this script, e.g. Sample

- Name of the primary shRNA library to which the sequencing reads should be aligned, e.g. LibraryName.

- Optional: additional names of other shRNA library names can be specified, in case your sequenced sample was derived from a cell population transduced with several shRNA libraries.

Example command line:

In [2]: run align_primary.py ../cropped/Sample.fa Sample LibraryName

44 The script generates several output files, most importantly a table of the frequencies with which each individual shRNA was detected in the sequencing data. In the above example, this file will be named SampleLibraryName.counts, located in the subdirectory counts. Once this counts file was successfully generated, files in the directories cropped and maps can be removed.

45 Repeat Steps 42-44 for all sequenced samples. Typically, these will consist of sets of the $t 0$ sample, a treated sample and an untreated sample. Corresponding example count files, named Example_Primary_t 0. counts,

Example_Primary_treated.counts and Example_Primary_untreated.counts, respectively, are provided in the subdirectory counts.

46 The results from the primary screen are analyzed using the script analyze_primary_screen.py with the following command-line arguments:

- Prefix for output files, e.g. ../results/Example

- Counts file from t0 sample, e.g. ../counts/Example_Primary_t0.counts

- Counts file from untreated sample, e.g. ../counts/

Example_Primary_untreated.counts

- Counts file from treated sample, e.g. ../counts/ Example_Primary_treated.counts

- File containing growth parameters G and K, e.g. ../growth/ Example_Primary_GK.txt

- Optional: information on negative controls. If this argument is not provided, the program interprets all shRNA names starting with the prefix " 0 " as negative controls. Alternatively, custom prefixes can be listed on the command line as consecutive arguments. If no negative control shRNAs are present in the library, the keyword "all" can be 
provided to use all shRNAs as an approximation of the "null distribution". This option is generally not recommended.

Example command line:

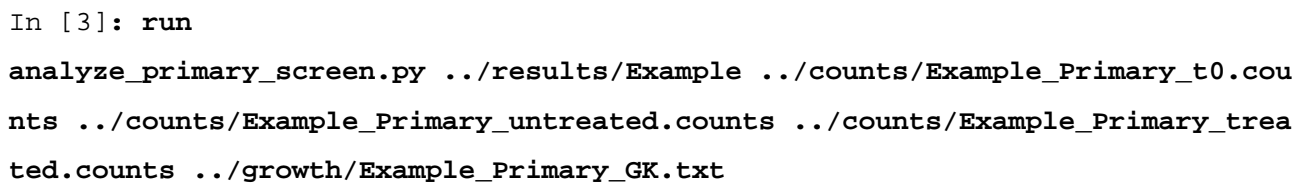

The script generates output on the terminal screen to summarize statistics of the input data. Example screen output:

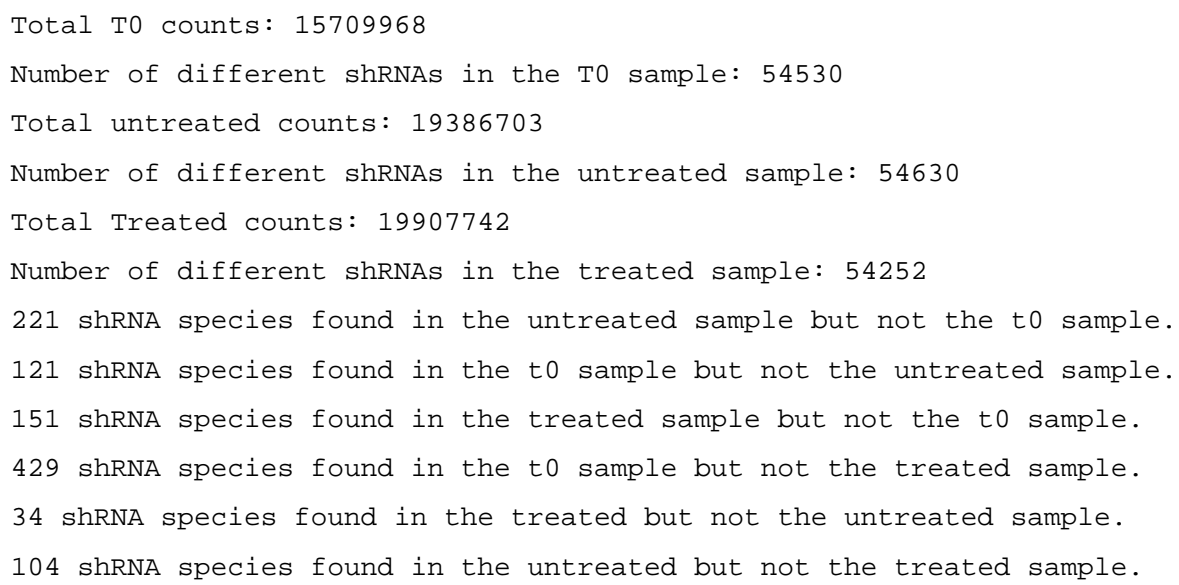

The script generates the following output files:

- Example.gammas:

Each line summarizes information for one shRNA with the following tabseparated fields: Name of shRNA, growth phenotype in the absence of treatment (gamma), counts in t0 sample, counts in untreated sample.

- Example.taus:

Each line summarizes information for one shRNA with the following tabseparated fields: Name of shRNA, growth phenotype in the presence of treatment (tau), counts in t0 sample, counts in treated sample.

- Example.rhos:

Each line summarizes information for one shRNA with the following tabseparated fields: Name of shRNA, phenotype quantifying the resistance to treatment (rho), counts in t0 sample, counts in treated sample.

- Example_pvalues.txt: 
Each gene one line with the following tab-separated fields:

- $\quad$ Entrez Gene ID

- Official Gene Symbol

- Gene information

- Number of shRNAs for which gammas could be calculated

- Gene scored as (P)rotective (i.e. shRNAs were enriched) or (S)ensitizing (i.e. shRNAs were depleted) based on Gamma phenotype

- $\quad$ P value for gene based on gamma phenotype, calculated using Mann-Whitney U test

- P value for gene based on gamma phenotype, calculated using Kolmogorov-Smirnov test

- Number of shRNAs for which taus could be calculated

- Gene scored as (P)rotective (i.e. shRNAs were enriched) or (S)ensitizing (i.e. shRNAs were depleted) based on tau phenotype

- $\quad$ P value for gene based on tau phenotype, calculated using MannWhitney U test

- $\quad$ P value for gene based on tau phenotype, calculated using Kolmogorov-Smirnov test

- Number of shRNAs for which rhos could be calculated

- Gene scored as (P)rotective (i.e. shRNAs were enriched) or (S)ensitizing (i.e. shRNAs were depleted) based on rho phenotype

- $\quad$ P value for gene based on rho phenotype, calculated using MannWhitney U test

- $\quad$ P value for gene based on rho phenotype, calculated using Kolmogorov-Smirnov test

This file is best opened in a program like Microsoft Excel to allow sorting of genes by $\mathrm{P}$ value.

We have previously provided mathematical definitions of the quantitative phenotypes (gamma, tau and rho) and of the approach for calculating $\mathrm{P}$ values ${ }^{9}$.

The script also generates the following graphical output in individual pop-up windows:

- $\quad$ Scatter plots of comparing shRNA frequencies in pairs of samples (e.g. Fig. 4a)

- Histograms of the number of shRNA phenotypes obtained for each gene (e.g. Supplementary Fig. 1a). 
- Comparison of the phenotypes of all shRNAs, compared to negative control shRNAs (either as cumulative curves, e.g. Supplementary Fig. 1b, or histograms, Fig. 4b)

- $\quad$ Scatter plots comparing gene P values calculated using the Mann-Whitney U (MW) test of the Kolmogorov-Smirnov (KS) test. The two tests generally yield very similar results (Supplementary Fig. 2a)

- Scatter plot comparing gene $\mathrm{P}$ values based on growth in the absence of treatment (gamma) with $\mathrm{P}$ values based on growth in the presence of treatment (tau) (Fig. 5a).

These graphs are saved as graphics file with the prefix specified on the command line. They can also be explored interactively, e.g. by zooming into areas of the $\mathrm{P}$ value scatter plots.

\section{? TROUBLESHOOTING}

47 After running the script, the following interactive functions can be called on the command line to explore the data interactively: gene_gamma(), gene_tau(), gene_rho().

The functions accept the following arguments:

- Name of gene of interest, in quotes. E.g. 'VPS54'.

- Optional: AllLabels (Boolean):

- True: Display names of shRNAs targeting the gene of interest

- False (default): Do not display these names

- Optional: MarkerSize (Integer):

- $\quad$ Size of circles representing shRNAs. Default: 40

- Optional: ShowLegend (Boolean):

- True: Display legend identifying shRNAs targeting the gene of interest and negative control shRNAs

- False (default): Do not display this legend

For example, the following command generates the graph shown in Fig. 5b:

In [4]: gene_rho('VPs54')

\section{Recloning individual shRNAs for validations and for the genetic interaction map - TIMING $4-5 d$}

48 Based on the results from the primary screen, choose hit genes to be included in validation experiments and in the genetic interaction map. For each gene, choose the 3-4 shRNAs that showed the strongest phenotypes - ideally in two or more independent experimental replicates of the primary screen. Also choose 12 or more negative control shRNAs. 
49 To clone these individual shRNAs into the barcoded vector pMK1200 (ref. 9) first digest overnight $1 \mu \mathrm{g}$ of plasmid DNA with $1 \mu \mathrm{BstXI}$ according to manufacturer's instructions, run the digestion reaction in a $0.8 \%(\mathrm{wt} / \mathrm{vol})$ agarose gel and extract using the gel extraction kit according to manufacturer's instructions.

CRITICAL STEP Do not dephosphorylate the vector backbone, since the insert will consist of unphosphorylated oligonucleotides.

50| Order top and bottom oligonucleotides corresponding to hit shRNA sequences formatted as in the example below for the target site TTTCTTACTCACCCTAAGAACT. Sequence in red represent unique overhangs that are compatible with BstXI-digested pMK1200; sequence in blue represent the shRNA loop sequence.

Top oligonucleotide:

CGTTCTTAGGGTGAGTAAGAAATAGTGAAGCCACAGATGTATTTCTTACTCACCCT AAGAACTTGCC

Bottom oligonucleotide:

AGTTCTTAGGGTGAGTAAGAAATACATCTGTGGCTTCACTATTTCTTACTCACCCTA AGAACGCGCT

51 Prepare oligonucleotide annealing mix:

\begin{tabular}{lcl}
\hline Compound & amount per sample & final concentration \\
\hline water & $23 \mathrm{ul}$ & \\
top oligo & $1 \mathrm{ul}(100 \mathrm{uM})$ & $2 \mathrm{uM}$ \\
bottom oligo & $1 \mathrm{ul}(100 \mathrm{uM})$ & $2 \mathrm{uM}$ \\
$2 \mathrm{X}$ annealing buffer & $25 \mathrm{ul}$ & $1 \mathrm{X}$ \\
\hline
\end{tabular}

52 Incubate annealing mix at $95^{\circ} \mathrm{C}$ for 5 min in PCR machine with heated lid, remove to bench and allow it to cool to room temperature for $10 \mathrm{~min}$.

53 Prepare 1:20 dilution of annealing mixture with $\mathrm{H}_{2} \mathrm{O}$

54 Assemble the following reaction for ligating the annealed oligonucleotides into the digested barcoding vector:

25 ng BstXI-digested, purified pMK1200

1 ul 1:10 diluted annealed oligonucleotides

1 ul 10X T4 DNA Ligase buffer

1 ul T4 DNA Ligase (2000 U/uL)

55 Ligate for $2 \mathrm{~h}$ at room temperature

56 Transform $2.5 \mu$ l ligation mixture into DH5a cells and plate onto LB plates containing $100 \mathrm{ug} / \mathrm{mL}$ carbenicillin. 
57 To validate correct shRNA inserts, sequence clones with primers 5'pSico-Ecoinsert-seq or 3' pSico-Pci-insert-seq (Table 1).

58 As shRNAs are individually cloned and barcoded as described above, they are catalogued in a tab-delimited text file called shRNAs.txt in the subdirectory LUT. An example of such a file is provided. In this file, each line summarizes information for one shRNA in the following tab-separated fields:

- Name of barcoded shRNA construct, in the following format: EntrezGeneID_GeneSymbol_Number

- $5^{\prime}$-to-3' sequence of top oligo used to clone this shRNA

- $\quad$ shRNA name in original pooled library

- Name of pooled library

- Plasmid number

- $\quad 5^{\prime}$-to-3' sequence of upstream barcode

- $\quad 5^{\prime}$-to-3' sequence of downstream barcode

59 When the barcodes of a new plasmid of an individually cloned shRNA are identified by sequencing, the scripts check_upstream_barcode.py and check_downstream_barcode.py are used to check whether the barcodes are acceptable in terms of their Hamming distance to the barcodes of all other previously accepted shRNA plasmids, and whether they are compatible with restriction enzyme digests at later stages in this protocol. The only commandline argument for check_upstream_barcode.py and check_downstream_barcode.py are the upstream and downstream barcode sequences, respectively, in $5^{\prime}$-to- $3^{\prime}$ direction. The evaluation of the candidate barcodes is provided as terminal output. For each shRNA construct, one acceptable clone needs to be identified. Usually, sequencing of 1-3 clones per construct is sufficient to identify an acceptable clone.

Examples:

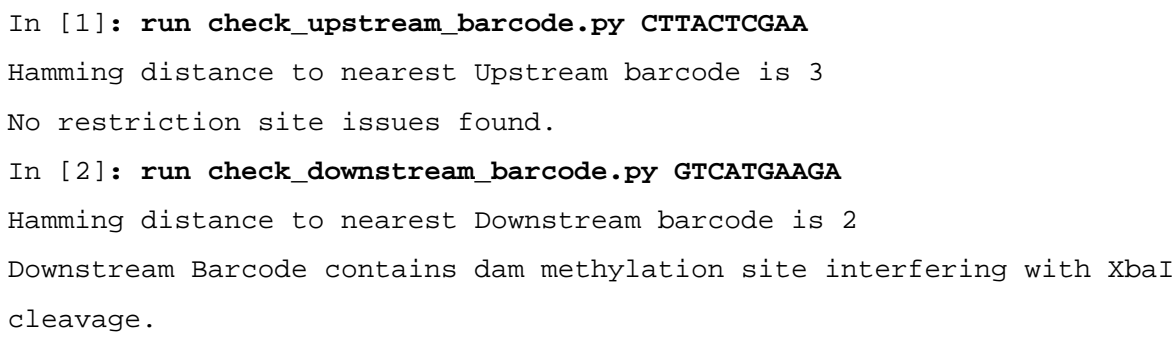

\section{Batch retest of individually cloned shRNAs - TIMING 1-21 d screen (depending on screen design) + 3 d sample preparation}

60 $\mid$ To validate the individually cloned shRNAs generated in Steps 48-59, pool them in equal quantities (e.g. $1 \mu \mathrm{g}$ of each) in a tube, and follow steps 1-38 with 
the following substitutions: (a) In Step 2, use the pool of individually cloned shRNAs instead of the library plasmid pool for transfection. (b) In Step 27, use one of the following forward primers: oMK371, oMK372, oMK373, oMK374, oMK375, oMK376, oMK377, or oMK378 (Table 1). For each sample that will be sequenced in the same lane on a flow cell, a different forward primer needs to be chosen to introduce a different index. For all samples, use the same reverse primer, oMCB922 (Table 1). (c) In Step 33, the expected PCR product size is $\sim 170$ bp. (d) In Step 38, no custom primer needs to be added to the Illumina primer mix.

\section{? TROUBLESHOOTING}

\section{Data analysis for the batch retest - TIMING $1 \mathrm{~d}$}

61 Move the sequencing results files, in fasta or Illumina fastq format, to the subdirectory sequencing.

62 On the command line, change to the directory scripts, and enter the IPython console with the following command:

ipython --pylab

The Enthought python distribution enables interactive graphical data display. If you do not want to use this feature, you can execute all scripts directly from the terminal command line by replacing "run" with "python" in the following command line examples (Steps 63-69). In that case, where graphical output is created as part of the program pipeline, the pop-up window with the graph needs to be closed for the program to proceed.

63 Run the script crop_reads.py to process the sequencing data with the following command-line arguments:

- Name of the sequencing data file, e.g. ../sequencing/

BatchRetestSample.fastq

- $\quad$ Name of the output file, e.g. ../cropped/BatchRetestSample.fa

- Number of bases after which the sequencing read should be truncated. For the analysis of batch retest samples, 16 is sufficient.

Example command line:

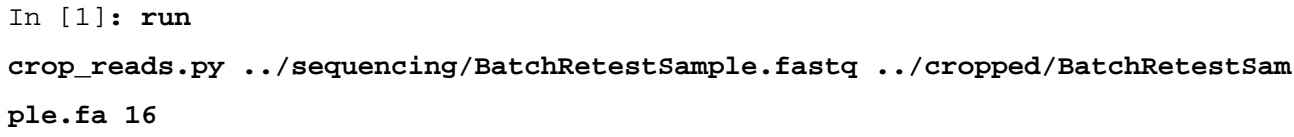

64 Run the script align_batch_retest.py to align the cropped reads to the shRNA library reference with the following command-line arguments:

- Cropped sequencing data file, e.g. ../cropped/BatchRetestSample.fa 
- Prefix for the output files generated by this script, e.g.

BatchRetestSample

Example command line:

In [2] : run align_batch_retest.py ../cropped/BatchRetestSample.fa
BatchRetestSample

65 The script generates a table of the frequencies with which each individual shRNA was detected in the sequencing data. In the above example, this file will be named BatchRetestSample.counts, located in the subdirectory counts. Once this counts file was successfully generated, files in the directory cropped can be removed.

66 $\mid$ Repeat Steps 63-65 for all sequenced samples. For batch retests, these may consist of one t0 sample, an untreated sample and several differentially treated samples. For each treatment condition, a file containing the $\mathrm{G}$ and $\mathrm{K}$ parameters must be created in the subdirectory growth, as described for primary screens (Step 39).

67 | To calculate gamma phenotypes, use the script batch_retest_shRNA_gammas.py with the following command-line arguments:

- $\quad$ Prefix for output files

- Counts file for t0 sample

- Counts file for untreated sample

- File with growth parameters $\mathrm{G}$ and $\mathrm{K}$ ( $\mathrm{K}$ is not used by this script)

- Optional: information on negative controls. If this argument is not provided, the program interprets all shRNA names starting with the prefix " 0 " as negative controls. Alternatively, custom prefixes can be listed on the command line as consecutive arguments. If no negative control shRNAs are present in the library, the keyword "all" can be provided to use all shRNAs as an approximation of the "null distribution". This option is generally not recommended.

This program creates the output file Prefix.gammas, in the same format as the gammas file generated by analyze_primary_screen.py, as well as several graphs corresponding to those generated by analyze_primary_screen.py.

68 To calculate tau phenotypes, use the script batch_retest_shRNA_taus.py, with the following command-line arguments:

- $\quad$ Prefix for output files

- Counts file for t0 sample

- Counts file for treated sample 
- File with growth parameters $\mathrm{G}$ and $\mathrm{K}$.

- Optional: information on negative controls. If this argument is not provided, the program interprets all shRNA names starting with the prefix " 0 " as negative controls. Alternatively, custom prefixes can be listed on the command line as consecutive arguments. If no negative control shRNAs are present in the library, the keyword "all" can be provided to use all shRNAs as an approximation of the "null distribution". This option is generally not recommended.

This program creates the output file Prefix.taus, in the same format as the taus file generated by analyze_primary_screen.py, as well as several graphs corresponding to those generated by analyze_primary_screen.py.

69| To calculate rho phenotypes, use the script batch_retest_shRNA_rhos.py, with the following command-line arguments:

- $\quad$ Prefix for output files

- Counts file for untreated sample

- Counts file for treated sample

- $\quad$ File with growth parameters $\mathrm{G}$ and $\mathrm{K}$.

- Optional: information on negative controls. If this argument is not provided, the program interprets all shRNA names starting with the prefix " 0 " as negative controls. Alternatively, custom prefixes can be listed on the command line as consecutive arguments. If no negative control shRNAs are present in the library, the keyword "all" can be provided to use all shRNAs as an approximation of the "null distribution". This option is generally not recommended.

This program creates the output file Prefix.rhos, in the same format as the rhos file generated by analyze_primary_screen.py, as well as several graphs corresponding to those generated by analyze_primary_screen.py.

Example command line:

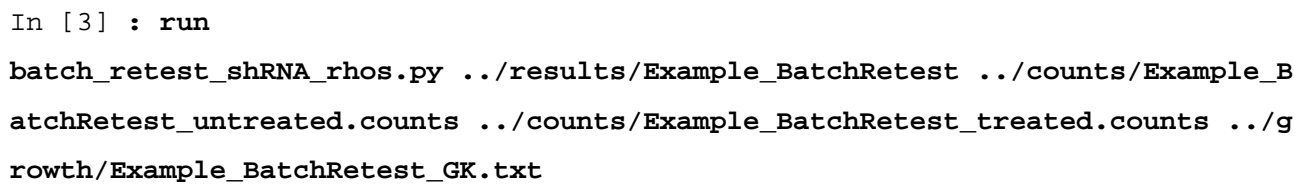

This command will create the output file ../results/Example_BatchRetest.rhos 


\section{Construction of double-shRNA libraries - TIMING 3 d}

70 | To construct double-shRNA libraries, select individually cloned shRNAs based on their performance in the batch retest. Only shRNAs that qualitatively reproduce the phenotype observed in the primary screen should be considered for the double-shRNA library. Select at least 3 shRNAs targeting each gene, and 12 or more negative control shRNAs. Pool the plasmids of these shRNAs in equal amounts in one tube, and split the pool to perform 2 restriction digests to generate the insert and backbone fragments. In one, digest $1 \mu \mathrm{g}$ of the pooled shRNAs with KpnI + AvrII, and purify a 1011 bp-long band (insert) from $0.8 \%$ (wt/vol) agarose gel using the Qiagen gel extraction kit according to the manufacturer's instructions. In the other, digest $1 \mu \mathrm{g}$ of the pooled shRNAs with $\mathrm{KpnI}+\mathrm{XbaI}$, and purify a 7808 bp-long band (backbone) from gel.

71 Ligate overnight at $16^{\circ} \mathrm{C}, 1 \mu \mathrm{g}$ of the $7808 \mathrm{bp}$-long fragment to $388 \mathrm{ng}$ of the 1011 bp-long fragment in $10 \mu \mathrm{l}$, using $1 \mu \mathrm{T} 4$ DNA ligase (2000 U) and $1 \mu \mathrm{l}$ 10X T4 DNA ligase buffer.

72 Transform $5 \mathrm{ul}$ of this mixture into MegaX bacterial cells by electroporation in a 1-mm gap cuvette at $2 \mathrm{kV}, 200 \mathrm{ohms}, 25 \mathrm{uF}$, and recover in $11 \mathrm{~mL} \mathrm{SOC}$ medium in Erlenmeyer flask $1 \mathrm{~h}$ at $37^{\circ} \mathrm{C}$ shaking at $250 \mathrm{rpm}$.

73 Plate bacteria on sufficient numbers of Qtray agar plates to get 1000 colonies per library shRNA. Typically, $500 \mathrm{uL}$ plated from the recipe above yields $\sim 100,000$ colonies per plate.

74 | Incubate overnight at $37^{\circ} \mathrm{C}$, scrape colonies using $20 \mathrm{~mL}$ LB per plate and a glass rod.

75 Collect LB from all plates, and proceed to Qiagen gigaprep instructions.

76 Prepare library DNA using Qiagen gigaprep kit; typical yield is $\sim 10 \mathrm{mg}$.

\section{Pooled screening of double-shRNA library - TIMING 1-21 d screen + 3 d sample preparation}

77 | To screen the double-shRNA library, follow steps 1-38 with the following substitutions: (a) In Step 2, use the double-shRNA library instead of the primary shRNA library for transfection. (b) In Step 27, use one of the following forward primers: oMK371, oMK372, oMK373, oMK374, oMK375, oMK376, oMK377, or oMK378 (Table 1). For each sample that will be sequenced in the same lane on a flow cell, a different forward primer needs to be chosen to introduce a different index. For all samples, use the same reverse primer, oMCB847 (Table 1). (c) In Step 33, the expected PCR product size is $\sim 190$ bp. (d) In Step 38, no custom primer needs to be added to the Illumina primer mix.

\section{? TROUBLESHOOTING}




\section{Data analysis double-shRNA screen, GI map construction - TIMING 4 d}

78 In subdirectory LUT, create a tab-delimited text file in which each line corresponds to the plasmid number of a plasmid included in the double-shRNA library. An example file, DoubleshRNA_plasmids.txt, is provided. If different plasmids are used in the first and second position, create one file for each set of plasmids.

79| Move the sequencing results files, in fasta or Illumina fastq format, to the subdirectory sequencing.

80 $\mid$ On the command line, change to the directory scripts, and enter the IPython console with the following command:

ipython --pylab

The Enthought python distribution enables interactive graphical data display. If you do not want to use this feature, you can execute all scripts directly from the terminal command line by replacing "run" with "python" in the following command line examples (Steps 81-93). In that case, where graphical output is created as part of the program pipeline, the pop-up window with the graph needs to be closed for the program to proceed.

81 Run the script crop_reads.py to process the sequencing data with the following command-line arguments:

- Name of the sequencing data file, e.g. ../sequencing/

DoubleshRNASample.fastq

- Name of the output file, e.g. ../cropped/DoubleshRNASample.fa

- Number of bases after which the sequencing read should be truncated. For the analysis of double shRNA samples, 26 is sufficient.

Example command line:

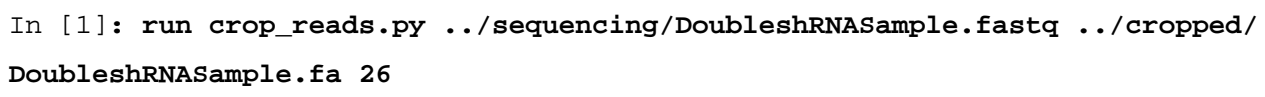

82 I Run the script align_double_shRNAs.py to align the cropped reads to the shRNA library reference with the following command-line arguments:

- $\quad$ Cropped sequencing data file, e.g. ../cropped/DoubleshRNASample.fa

- Prefix for the output files generated by this script, e.g. DoubleshRNASample

Example command line: 
In [2]: run align_double_shRNAs.py ../cropped/DoubleshRNASample.fa

DoubleshRNASample

83 The script generates several output files, most importantly a table of the frequencies with which each double-shRNA was detected in the sequencing data. In the above example, this file will be named

DoubleshRNASample.counts, located in the subdirectory counts. Once this counts file was successfully generated, files in the directory cropped can be removed.

84 Steps 81-83 are repeated for all sequenced samples. For double-shRNA screens, these typically consist of one t0 sample, a treated sample and an untreated sample. Two replicates of the screen should typically be carried out. The following example count files are provided in the subdirectory counts: Rep1_t0.counts, Rep1_treated.counts, Rep1_untreated.counts, Rep2_t0.counts, Rep2_treated.counts, Rep2_untreated.counts.

85 For each treatment condition, a file containing the $\mathrm{G}$ and $\mathrm{K}$ parameters must be created in the subdirectory growth, as described for primary screens (Step 39). Example files Rep1_GK.txt and Rep2_GK.txt are provided.

86 The script double_shRNA_phenotypes.py is called with only one command-line argument, the prefix for the output files, e.g. ../results/Rep1. The remaining is supplied via an interactive dialogue in the terminal window.

Example:

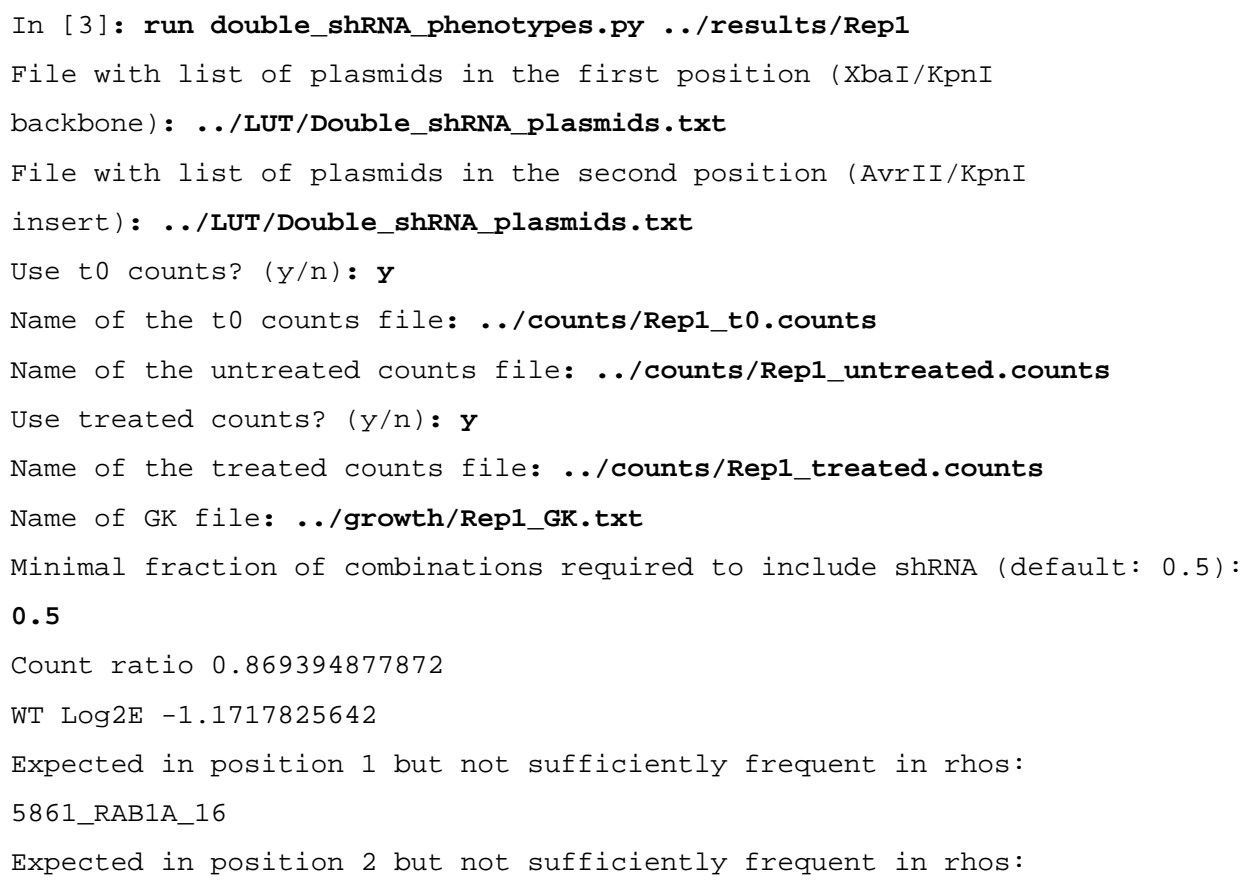




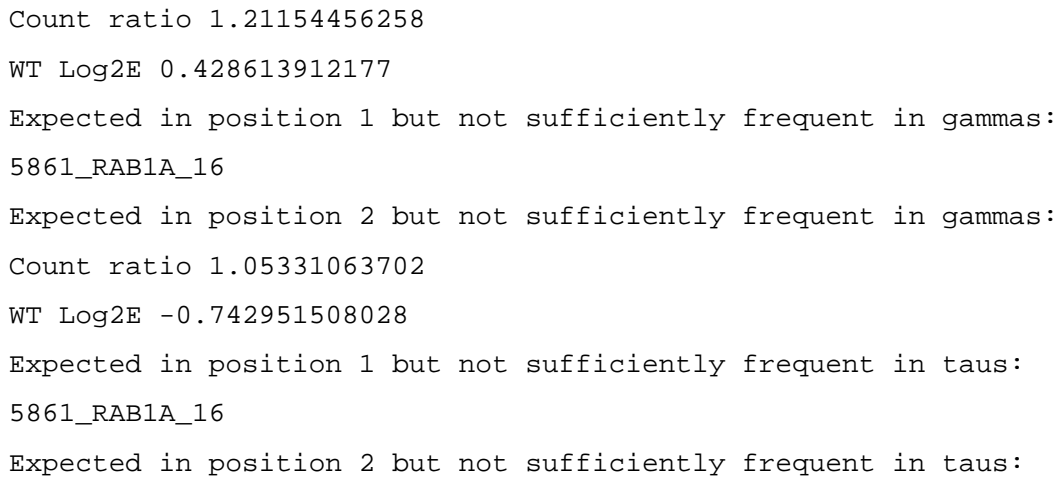

The script generates several graphs. These graphs analyze the phenotypes of pairs of shRNAs, $\mathrm{A}$ and $\mathrm{B}$ in the $\mathrm{AB}$ and the $\mathrm{BA}$ orientations. $\mathrm{AB}$ and $\mathrm{BA}$ phenotypes should be very similar; large differences between $\mathrm{AB}$ and $\mathrm{BA}$ are therefore mostly reflective of experimental noise. The distribution of $\mathrm{AB}-\mathrm{BA}$ differences in the entire dataset is visualized by a histogram (e.g. Supplementary Fig. 3a). Two-dimensional histograms (based on hexagonally binned heatmaps) explore the dependence of noise on phenotypic strength (Fig. Supplementary Fig. 3b) and abundance of double shRNAs at time t0 (Fig. 6). Less abundant double-shRNAs typically give noisier phenotypes, indicating that data quality can be further improved by scaling up the cell population for the pooled screen. A further internal quality control is the comparison of phenotypes of double-shRNAs containing one shRNA targeting a hit gene and one negative control shRNA in the two possible orientations (Supplementary Fig. 4). A small systematic deviation is observed since shRNAs in the second position of the double-shRNAs are slightly more effective.

The script also generates several output files, which are used by scripts in subsequent steps.

\section{? TROUBLESHOOTING}

87 Genetic interactions are calculated by the script calculate_GIs.py. The only command line argument is the same Prefix entered for the previous script, e.g. ../ results/Rep1. In an interactive dialogue, the phenotypes for which genetic interactions should be calculated are specified.

Example:

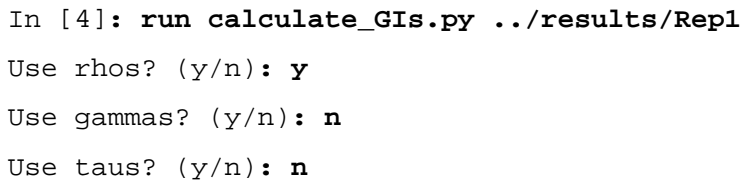

The script generates several graphs in pop-up windows:

- Comparison of different definitions for the expected double-mutant phenotype. Orange line: Sum definition, green line: Product definition, blue: empirical linear fit for each shRNA (dark blue dots indicate the average phenotypes and slopes for 
shRNAs targeting a given gene, light blue error bars indicate standard deviation) (Supplementary Fig. 5a)

- Comparison of slopes for empirical linear fits for shRNAs obtained either along "rows" or "columns" of the double-shRNA phenotype table - both should be identical. This graph can reveal noise or systematic biases. (Supplementary Fig. $5 b)$.

88 After running the script, several interactive functions can be called on the command line to explore the data interactively:

- $\quad$ rho_sd(), gamma_sd(). Comparison of single shRNA phenotypes (derived from double shRNAs containing one negative control shRNAs) and double shRNA phenotypes, in combination with one shRNA of interest.

These functions accept the following arguments:

- Name of an shRNA of interest, in quotes. E.g. 'SEC23B_4'.

- Optional: LabelAboveZ (floating point number): Display names of shRNAs if their genetic interaction with the shRNA of interest is this many standard deviations above the mean. Default is 100 , which typically suppresses all shRNA labels

- Optional: Link (Boolean):

$\checkmark \quad$ True (default): Link points representing the same shRNA in the first and second positions of a double-shRNA by a line.

$\checkmark$ False: Do not display this line

For example, the following command generates the graph shown in Supplementary Fig. 6a:

In [5]: rho_sd('SEC23B_4',

LabelAboveZ $=2.5$, Link=True)

- -gene_rho_sd(), gene_gamma_sd(). Similar to the previous function, except that data from shRNAs targeting the same gene are averaged. This typically vastly reduces the noise.

These functions accept the following arguments:

- Gene of interest, in quotes. E.g. 'SEC23B'.

- Optional: LabelAboveZ (floating point number): 
Display names of genes if their average genetic interaction with the gene of interest is this many standard deviations above the mean. Default is 100 , which typically suppresses all shRNA labels

- Optional: Link (Boolean):

$\checkmark \quad$ True (default): Link points representing the same gene in the first and second positions of a double-shRNA by a line.

$\checkmark$ False: Do not display this line

- Optional: PlotSD (Boolean):

- True (default): Show error bars representing the standard deviation of the values for individual shRNAs targeting the gene of interest.

False: Do not display error bars.

For example, the following command generates the graph shown in Supplementary Fig. 6b:

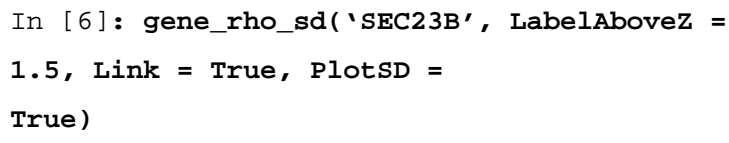

- ab_rho_sd_yellow_blue() and ab_gamma_sd_yellow_blue(). Similar to rho_sd() and gamma_sd(), but data for each shRNA are averaged for the shRNA occurring in the first and in the second position within the double-shRNA.

These functions accept the following arguments:

- Name of an shRNA of interest, in quotes. E.g. 'SEC23B_4'.

- Optional: ShowLabels (Boolean):

$\checkmark \quad$ True: Display shRNA names.

$\checkmark \quad$ False (default): Do not display shRNA names.

For example, the following command generates the graph shown in Fig. 7:

In $[7]:$ 
ab_rho_sd_yellow_blue ( 'SEC23B_4', ShowLabels=True

)

89| The script also generates several output files, which are used by scripts in subsequent steps. These include tables of genetic interactions calculated according to the different definitions we described previously ${ }^{9}$. If none of these definitions are a good fit for a given experiment, users can implement additional custom definitions by editing the script calculate_GIs.py.

90| Repeat Steps 86-89 for the second replicate of the screen.

91 The script compare_GIs.py averages experimental replicates and compares different definitions of genetic interactions. The two command-line arguments are:

- $\quad$ Prefix for the first replicate, e.g. ../results/Rep1

- $\quad$ Prefix for the first replicate, e.g. ../results/Rep2

The phenotypes (rhos, gammas, taus) for which genetic interactions should be compared are specified interactively. Example:

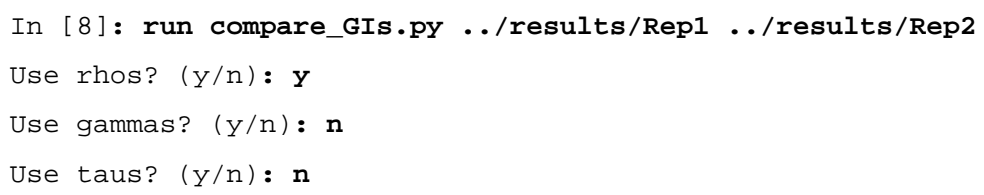

The script generates several output files that tabulate genetic interactions averaged between the two replicates. Different methods for calculating genetic interactions are indicated by the following code, which appears in the corresponding file names: Phenotype Expected Flip.

Phenotype can be Rho, Gamma or Tau. Expected can be $\mathrm{p}$ (for product definition of expected double-shRNA phenotypes), $\mathrm{s}$ (for sum definition of expected double-shRNA phenotypes), or $\mathrm{f}$ (for empirical fit definition of expected double-shRNA phenotypes). The code for Flip can be absent for raw genetic interactions, flip1 for Definition 1 of buffering/ synergistic genetic interactions, or flip2 for Definition 2 of buffering/synergistic genetic interactions. We have previously defined these different methods to calculate genetic interactions quantitatively ${ }^{9}$. For example, the above command line will create, among others, the following output file: ../results/Rep1Rep2_Rhofflip2GI.txt.

The script also generates several graphs as pop-up windows:

- Two-dimensional histograms (based on hexagonally binned heatmaps) visualize the correlation of genetic interactions between shRNA pairs across the two replicates (e.g. Supplementary Fig. 7a)

- Correlation coefficients for genetic interactions across the two replicates are summarized in a bar graph for the different methods for calculating genetic interactions (Supplementary Fig. 7b) 
- Mean genetic interaction values are summarized in a bar graph for genetic interactions calculated using the different methods and averaged between replicates (Supplementary Fig. 7c).

- Comparison of the means of Pearson correlation between the genetic interaction patterns of shRNAs targeting the same gene ("Intra-gene correlation") or different genes ("Inter-Gene correlation"), for genetic interactions calculated using the different methods and averaged between replicates (Supplementary Fig. 7d). For an appropriate definition of genetic interactions, the mean intra-gene correlation should be much larger than the mean inter-gene correlation.

92 Based on the results produced by the scripts calculate_GIs.py and compare_GIs.py, users should decide which definition of genetic interactions (as defined in Step 91, e.g. Rhof) is most appropriate for their data. Criteria for this decision are as follows:

- For a given shRNA, most double-shRNAs should show the expected double-shRNA phenotype according to the chosen definition. For example, Fig. 12 reveals that the sum and product definitions are not adequate for most genes in the example data set, therefore the empirical fit for individual shRNAs should be selected here.

- Genetic interaction patterns for shRNAs targeting the same gene should on average be more correlated than genetic interaction patterns for other shRNAs (Supplementary Fig. 7d).

93 The script filter_GIs.py aims to remove shRNAs with possible off-target effects from the dataset. Such shRNAs are detected as shRNAs whose genetic interaction pattern is not sufficiently correlated with that of other shRNAs targeting the same gene ${ }^{9}$. The script requires two command-line arguments:

- Genetic interaction file to be used as input (e.g. ../results/ Rep1Rep2_RhofGI.txt)

- Cutoff for required correlation of genetic interaction pattern with that of other shRNAs targeting the same gene, expressed in standard deviations of the distribution of correlation coefficients between genetic interaction patterns of a given shRNA and all other shRNAs in the data set (e.g. 0.8)

Example command line:

In [9]: run filter_GIs.py ../results/Rep1Rep2_RhofGI 0.8

The script lists the shRNAs that were removed from the dataset as screen output, for example:

Rejected shRNAs: [ 'C17orf75_3', 'CCT7_2', 'CCT7_3', 'COPB1_1', 'COPB1_2', 


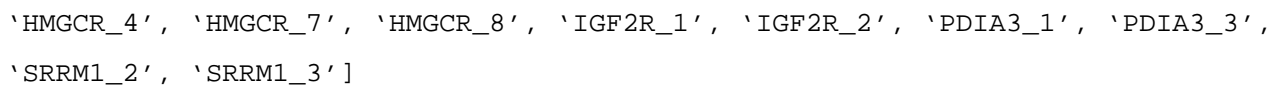

Two filtered genetic interaction files are generated, one with the shRNA-based genetic interactions (e.g. ../results/Rep1Rep2_KfGI_minZ0.8.txt), and one with gene-averaged genetic interactions, for which genetic interactions of shRNAs targeting the same gene are averaged (e.g. ../results/Rep1Rep2_KfGI_minZ0.8_GeneGI.txt).

A heatmap in a popup window visualizes the frequency of different outcomes for the shRNAs targeting a given gene (Supplementary Fig. 8). Only genes targeted by at least two correlating shRNAs are included in the gene-based genetic interaction map.

94 Open Cluster 3.0 (ref. 27) and load the gene-based genetic interaction file generated by the script filter_GIs.py (e.g. ../results/ Rep1Rep2_KfGI_minZ0.8_GeneGI.txt). Apply Hierarchical clustering on both "Genes" and "Arrays" using Uncentered Correlation as similarity metric and Average Linkage as clustering method. This will generate three files with the same prefix and the extensions .atr, .cdt and .gtr.

95 Open Java Tree View ${ }^{29}$ and load the .cdt file generated by Cluster 3.0 (e.g. ../ results/Rep1Rep2_KfGI_minZ0.8_GeneGI.txt.cdt) for interactive exploration of the genetic interaction map. Refer to the Java Tree Documentation (http:// jtreeview.sourceforge.net/manual.html) for instructions.

\section{? TROUBLESHOOTING}

Troubleshooting advice can be found in Table 2 .

\section{TIMING}

Steps 1-7, lentivirus preparation and infection: $7-10 \mathrm{~d}$

Steps $8-10$, conducting a screen: $1-21 \mathrm{~d}$

Steps 11-38, preparing samples for deep sequencing: $3 \mathrm{~d}$

Steps $39-47$, data analysis primary screen: $1-4 \mathrm{~d}$

Steps 48-59, recloning individual shRNAs: $4-5 \mathrm{~d}$

Step 60, batch retest of individually cloned shRNAs: $1-21 \mathrm{~d}$ screen $+3 \mathrm{~d}$ sample preparation

Steps 61-69, data analysis batch retest: $1 \mathrm{~d}$

Steps 70-76, construction of double-shRNA library: $3 \mathrm{~d}$

Step 77, pooled screening of double-shRNA library: 1-21 d screen $+3 \mathrm{~d}$ sample preparation

Steps 78-95, data analysis double-shRNA screen, GI map construction: $4 \mathrm{~d}$ 


\section{ANTICIPATED RESULTS}

The results of the primary screen depend strongly on the biological question. In some cases, a single gene is detected as an exceptionally strong hit ${ }^{30}$. In other cases, dozens or hundreds of hit genes are detected ${ }^{10}$. In the latter case, a genetic interaction map is likely to generate hypotheses for functional connections between genes, such as physical interaction or enzyme-substrate relationships (Fig. 8). These can then be tested using classical cell biological and biochemical approaches.

\section{Supplementary Material}

Refer to Web version on PubMed Central for supplementary material.

\section{Acknowledgments}

We thank Emily LeProust and Siyuan Chen of Agilent Technologies for oligonucleotide libraries, Yuwen Chen and Shuyi Wang for technical assistance and feedback on the protocol, Max Horlbeck for testing pMK1200, Yuwen Chen, Max Horlbeck and Luke Gilbert for sharing gel images, Sean Collins for helpful discussions on data analysis. M.K. was funded by fellowships from the Jane Coffin Childs Memorial Fund for Medical Research and the University of California, San Francisco Program for Breakthrough Biomedical Research. M.C.B. was funded by a fellowship from the Leukemia and Lymphoma Society. J.S.W. was funded by the Howard Hughes Medical Institute, National Institutes of Health Grant 1U01CA168370-01, and a Howard Hughes Collaborative Initiative Award.

\section{References}

1. Bandyopadhyay S, et al. Rewiring of genetic networks in response to DNA damage. Science. 2010; 330:1385-9. [PubMed: 21127252]

2. Frost A, et al. Functional repurposing revealed by comparing S. pombe and S. cerevisiae genetic interactions. Cell. 2012; 149:1339-52. [PubMed: 22682253]

3. Jonikas MC, et al. Comprehensive characterization of genes required for protein folding in the endoplasmic reticulum. Science. 2009; 323:1693-7. [PubMed: 19325107]

4. Roguev A, et al. Conservation and rewiring of functional modules revealed by an epistasis map in fission yeast. Science. 2008; 322:405-10. [PubMed: 18818364]

5. Schuldiner M, et al. Exploration of the function and organization of the yeast early secretory pathway through an epistatic miniarray profile. Cell. 2005; 123:507-19. [PubMed: 16269340]

6. Tong AH, et al. Systematic genetic analysis with ordered arrays of yeast deletion mutants. Science. 2001; 294:2364-8. [PubMed: 11743205]

7. Tong AH, et al. Global mapping of the yeast genetic interaction network. Science. 2004; 303:80813. [PubMed: 14764870]

8. Collins SR, et al. Functional dissection of protein complexes involved in yeast chromosome biology using a genetic interaction map. Nature. 2007; 446:806-10. [PubMed: 17314980]

9. Kampmann M, Bassik MC, Weissman JS. Integrated platform for genome-wide screening and construction of high-density genetic interaction maps in mammalian cells. Proc Natl Acad Sci U S A. 2013; 110:E2317-26. [PubMed: 23739767]

10. Bassik MC, et al. A systematic mammalian genetic interaction map reveals pathways underlying ricin susceptibility. Cell. 2013; 152:909-22. [PubMed: 23394947]

11. Laufer C, Fischer B, Billmann M, Huber W, Boutros M. Mapping genetic interactions in human cancer cells with RNAi and multiparametric phenotyping. Nat Methods. 2013; 10:427-31. [PubMed: 23563794]

12. Roguev A, et al. Quantitative genetic-interaction mapping in mammalian cells. Nat Methods. 2013; 10:432-7. [PubMed: 23407553] 
13. Bassik MC, et al. Rapid creation and quantitative monitoring of high coverage shRNA libraries. Nat Methods. 2009; 6:443-5. [PubMed: 19448642]

14. Kaelin WG Jr. Molecular biology. Use and abuse of RNAi to study mammalian gene function. Science. 2012; 337:421-2. [PubMed: 22837515]

15. Cheung HW, et al. Systematic investigation of genetic vulnerabilities across cancer cell lines reveals lineage-specific dependencies in ovarian cancer. Proc Natl Acad Sci U S A. 2011; 108:12372-7. [PubMed: 21746896]

16. Luo B, et al. Highly parallel identification of essential genes in cancer cells. Proc Natl Acad Sci U S A. 2008; 105:20380-5. [PubMed: 19091943]

17. Luo J, et al. A genome-wide RNAi screen identifies multiple synthetic lethal interactions with the Ras oncogene. Cell. 2009; 137:835-48. [PubMed: 19490893]

18. Zuber J, et al. Toolkit for evaluating genes required for proliferation and survival using tetracycline-regulated RNAi. Nat Biotechnol. 2011; 29:79-83. [PubMed: 21131983]

19. Qi LS, et al. Repurposing CRISPR as an RNA-guided platform for sequence-specific control of gene expression. Cell. 2013; 152:1173-83. [PubMed: 23452860]

20. Gilbert LA, et al. CRISPR-mediated modular RNA-guided regulation of transcription in eukaryotes. Cell. 2013; 154:442-51. [PubMed: 23849981]

21. Wang T, Wei JJ, Sabatini DM, Lander ES. Genetic screens in human cells using the CRISPR-Cas9 system. Science. 2013; 343:80-4. [PubMed: 24336569]

22. Shalem O, et al. Genome-scale CRISPR-Cas9 knockout screening in human cells. Science. 2013; 343:84-7. [PubMed: 24336571]

23. Meerbrey KL, et al. The pINDUCER lentiviral toolkit for inducible RNA interference in vitro and in vivo. Proc Natl Acad Sci U S A. 2011; 108:3665-70. [PubMed: 21307310]

24. Silva JM, et al. Profiling essential genes in human mammary cells by multiplex RNAi screening. Science. 2008; 319:617-20. [PubMed: 18239125]

25. Dull T, et al. A third-generation lentivirus vector with a conditional packaging system. J Virol. 1998; 72:8463-71. [PubMed: 9765382]

26. Naldini $\mathrm{L}$, et al. In vivo gene delivery and stable transduction of nondividing cells by a lentiviral vector. Science. 1996; 272:263-7. [PubMed: 8602510]

27. Langmead B, Trapnell C, Pop M, Salzberg SL. Ultrafast and memory-efficient alignment of short DNA sequences to the human genome. Genome Biol. 2009; 10:R25. [PubMed: 19261174]

28. Eisen MB, Spellman PT, Brown PO, Botstein D. Cluster analysis and display of genome-wide expression patterns. Proc Natl Acad Sci U S A. 1998; 95:14863-8. [PubMed: 9843981]

29. Saldanha AJ. Java Treeview--extensible visualization of microarray data. Bioinformatics. 2004; 20:3246-8. [PubMed: 15180930]

30. Matheny CJ, et al. Next-Generation NAMPT Inhibitors Identified by Sequential High-Throughput Phenotypic Chemical and Functional Genomic Screens. Chem Biol. 2013; 20:1352-63. [PubMed: 24183972] 
a PRIMARY SCREEN

Genome wide shRNA library in pMK1047 vector

Package into lentivirus, infect cells

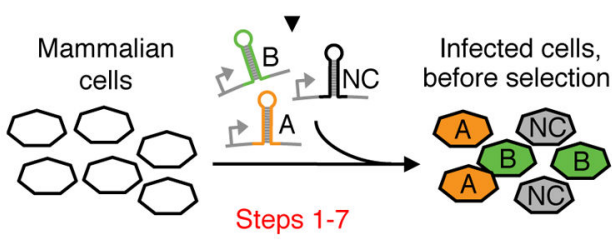

Steps 8-10

Selection based

on growth or physical separation

Selected

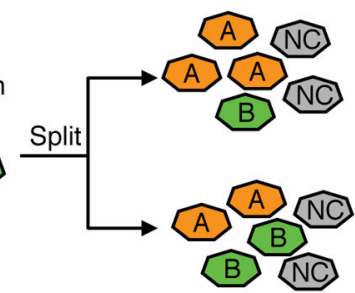

Unselected $\therefore$ shRNAs targeting gene $A$

- Negative control shRNAs

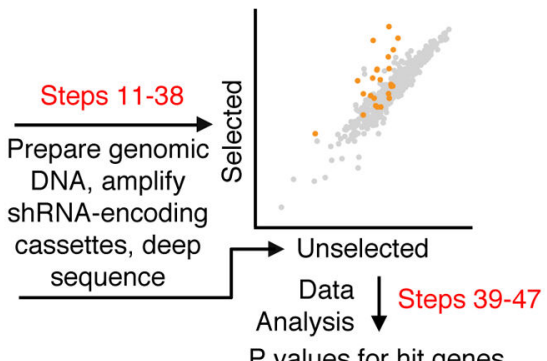

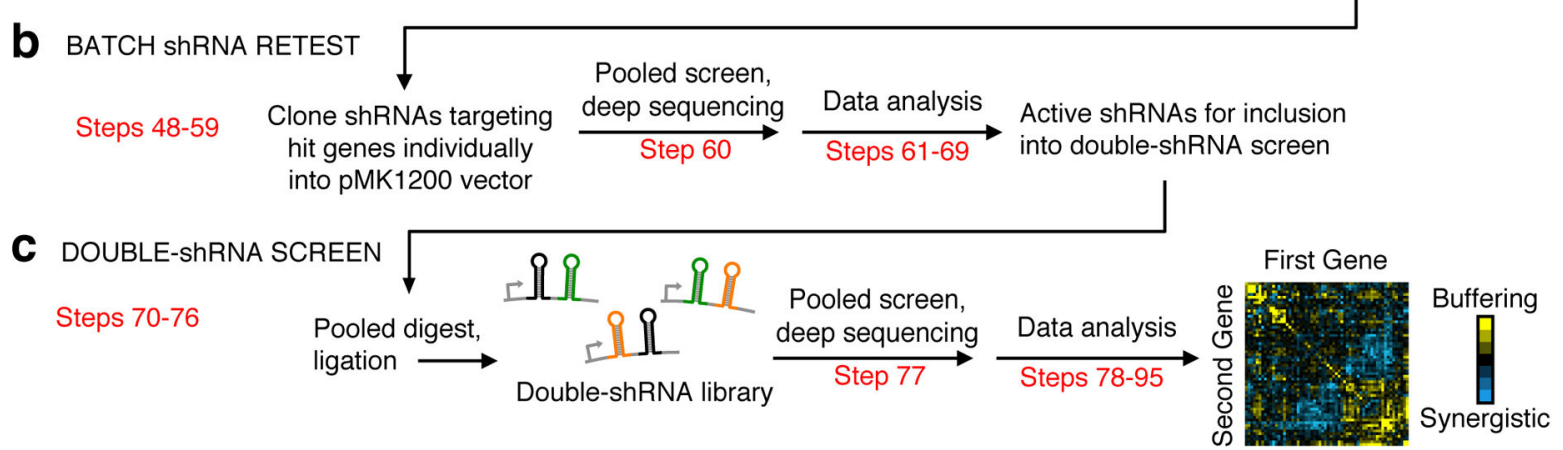

High-density quantitative GI map

Figure 1.

Overview of the experimental procedure. (a) Primary genome-wide shRNA screen, in which hit genes are identified. (b) Cloning of individual shRNAs against hit genes from the primary screen, and confirmation of their phenotype in a batch retest. (c) Construction and screening of a double-shRNA library representing all pairwise combination of selected shRNAs from the batch retest, and calculation of a systematic genetic interaction map. 
a GENOME-WIDE POOLED ShRNA LIBRARY

pMK1047

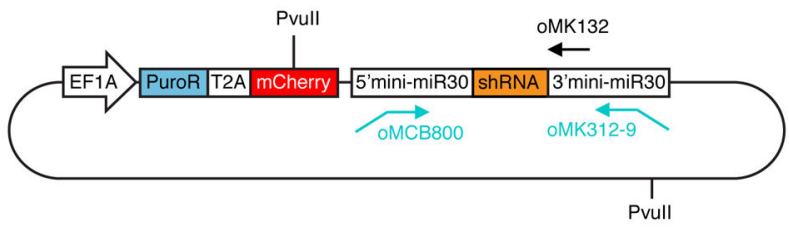

b INDIVIDUALLY BARCODED ShRNAs

pMK1200
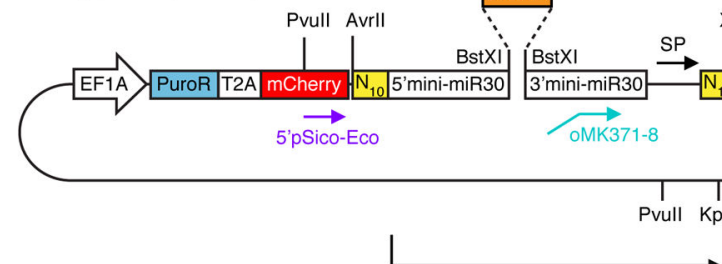

Pooled digest: - Avrll + Kpnl for insert

- Xbal + Kpnl for backbone

C DOUBLE-ShRNA LIBRARY

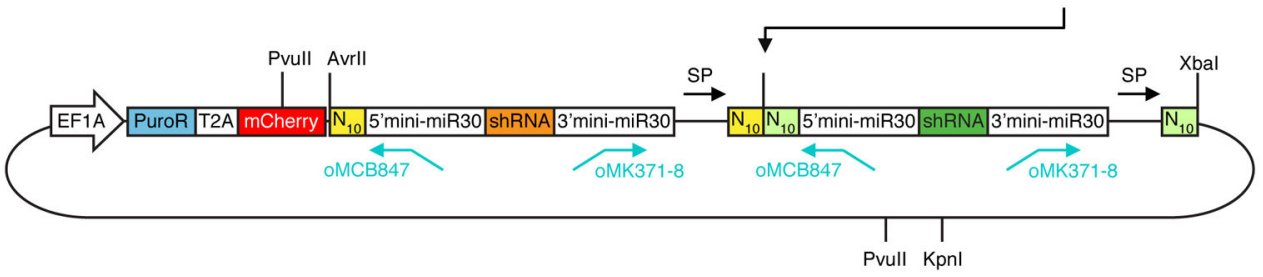

Figure 2.

Lentiviral plasmids used in the experimental procedure (not drawn to scale). The EF1A promoter drives expression of an mRNA that encodes a puromycin resistance marker, an mCherry fluorescent marker, and either one or two shRNAs embedded in a minimal miR-30 context. (a) Vector pMK1047 contains the pooled, ultracomplex genome-wide shRNA library. The shRNA expression cassette is flanked by PvuII sites that are 1,122 bp apart; this restriction enzyme is used to size-fractionate genomic DNA from cell populations transduced with pMK1047. Primers oMCB800 and one of the primers oMK312 or oMK313 (shown in turquoise; sequences in Table 1) are used to amplify the shRNA cassette from genomic DNA, and primer oMK132 is used as primer for Illumina sequencing to identify the shRNA, starting with the guide strand. (b) Vector pMK1200 is used to clone individual shRNAs. This vector is a pool of plasmids with random $\mathrm{N}_{10}$ barcodes upstream and downstream of the minimal miR-30 context. Annealed oligonucleotides encoding the shRNA of interest are ligated into BstXI-cut pMK1200. The combinations of barcodes and shRNA inserts are identified by sequencing with primers $5^{\prime}$ pSico-Eco or $3^{\prime}$ pSico-Pci (shown in purple; sequences in Table 1). The shRNA expression cassette is flanked by PvuII sites that are 1,177 bp apart. Primers oMCB922 and one of the primers oMK371 or oMK378 (shown in turquoise; sequences in Table 1) are used to amplify the shRNA cassette from genomic DNA, and the Illumina genomic sequencing primer (shown as SP) is used as primer for Illumina sequencing to identify the downstream barcode. (c) A double-shRNA library is created by digesting a pool of barcoded shRNA cloned in the pMK1200 backbone. This pool is digested with AvrII $+\mathrm{KpnI}$ to obtain an insert and with $\mathrm{XbaI}+\mathrm{KpnI}$ to obtain a 
backbone, the ligation of which results in a double-shRNA library. The double-shRNA expression cassette is flanked by PvuII sites that are 1,369 bp apart. Primers oMCB847 and one of the primers oMK371 or oMK378 (shown in turquoise; sequences in Table 1) are used to amplify the barcode junction portion from genomic DNA. Note that although both primers will anneal to two places within the double-shRNA cassette, only one amplicon will be generated by PCR. The Illumina genomic sequencing primer (shown as SP) is used as primer for Illumina sequencing to identify the combinatorial barcode. 

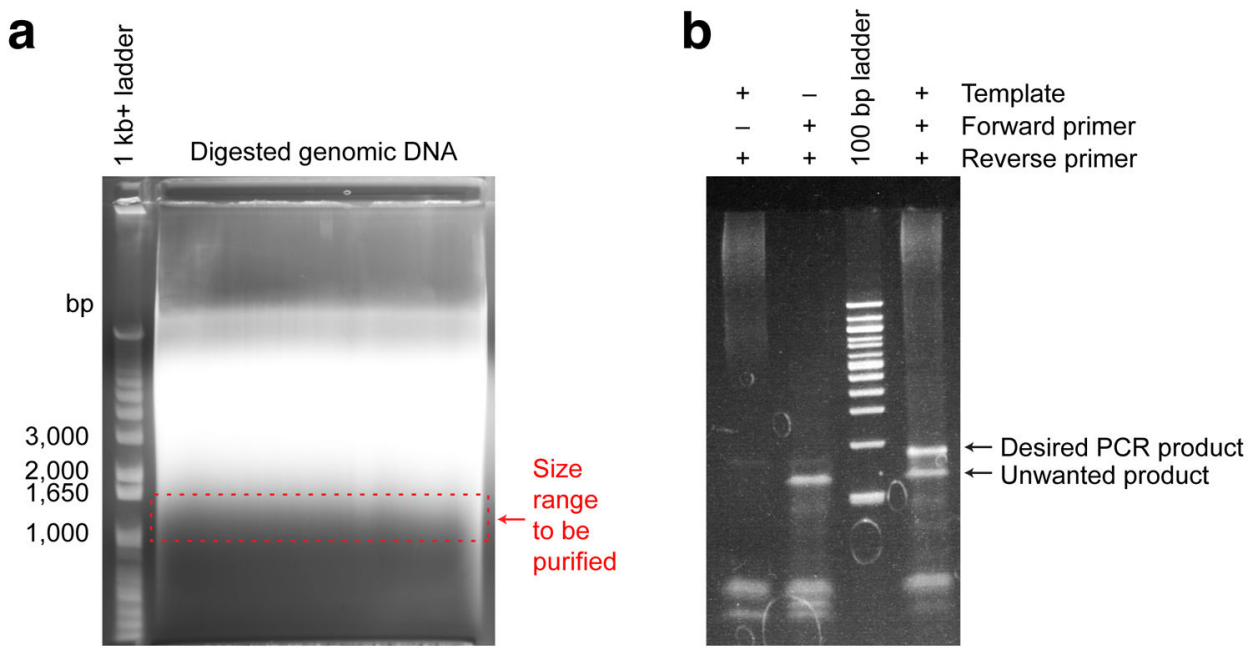

Figure 3.

Gel purification steps during sample preparation. (a) Size fractionation of genomic DNA. Genomic DNA isolated from a screen cell population is digested with PvuII and the size range between 1,000 and $1,650 \mathrm{bp}$, which contains the shRNA expressing cassette for all constructs shown in Figure 2, is excised from an ethidium bromide-stained $0.8 \%$ agarose gel, as indicated by the dashed red box, and purified for further use as a PCR template. (b) PCR products are separated on a $20 \%$ polyacrylamide gel and visualized by ethidium bromide staining. In addition to the desired PCR product (in the example shown a $190 \mathrm{bp}$ long product amplified from genomic DNA of cells transduced with a double-shRNA library), shorter products result from long primer dimers (the unwanted band is present in the control reaction lacking template, but not the control reaction lacking reverse primer). 

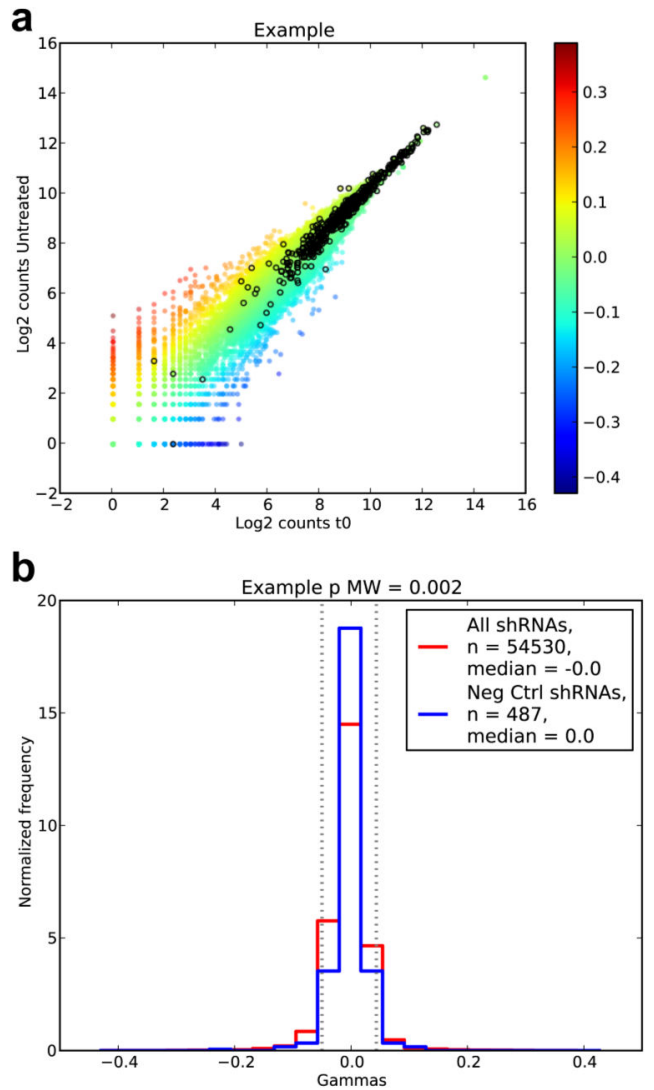

Figure 4.

Graphical output of the script analyze_primary_screen.py (screenshot). (a) Each dot represents one shRNA. X-axis: $\log _{2}$-transformed deep-sequencing counts of cells expressing this shRNA in the t0 population. Y-axis: $\log _{2}$-transformed deep-sequencing counts of cells expressing this shRNA in the untreated population at the endpoint of the experiment. Black circles represent negative-control shRNAs. Colored circles represent shRNAs designed to target genes; they are colored according to a heatmap representing the growth phenotype (gamma). (b) Distribution of growth phenotypes (gammas) for negative control shRNAs (blue dots) and shRNAs designed to target genes (red dots). Grey dashed lines indicate the range of phenotypes containing the middle $95 \%$ of negative control shRNAs. 

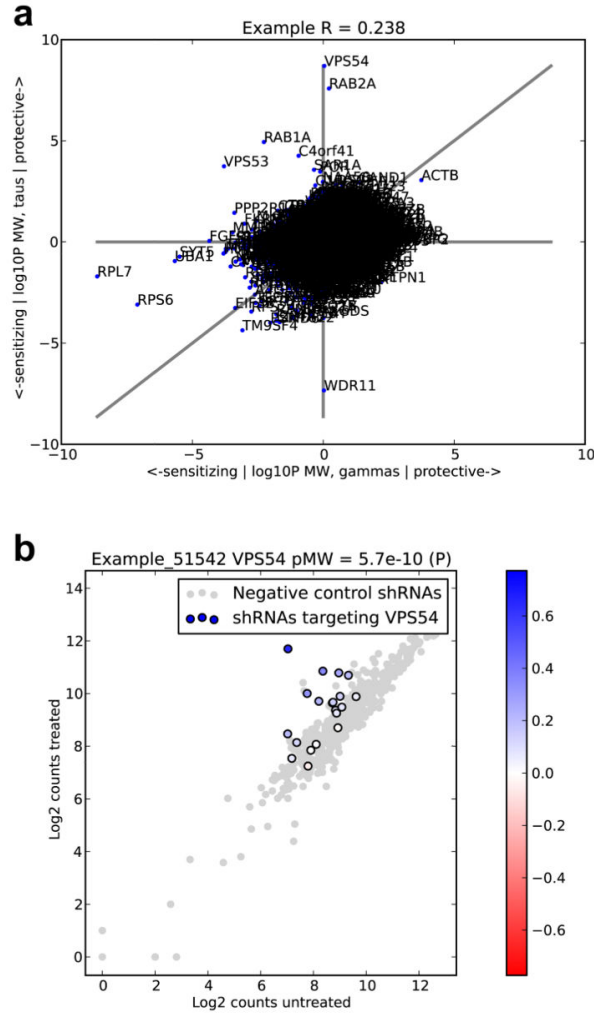

Figure 5 .

Graphical output of the script analyze_primary_screen.py (screenshot) (a) Each dot represents one gene. $\log _{10}$-transformed $\mathrm{P}$ values for an effect caused by gene knockdown were calculated by the Mann-Whitney test. Negative values indicate depletion of cells expressing shRNAs targeting the gene of interest from the population, positive values indicate enrichment. $\mathrm{X}$ axis: untreated cell population, $\mathrm{Y}$ axis: treated cell population. (b) This graph was generated interactively using the gene_rho() command after running the script (Step X). Each dot represents an shRNA. X-axis: $\log _{2}$-transformed deep-sequencing counts of cells expressing this shRNA in the untreated population at the endpoint of the experiment. Y-axis: $\log _{2}$-transformed deep-sequencing counts of cells expressing this shRNA in the treated population at the endpoint of the experiment. Grey semi-transparent dots represent negative control shRNAs. Solidly colored dots represent shRNAs targeting the gene VPS54; they are colored according to a heatmap representing the resistance phenotype (rho). 


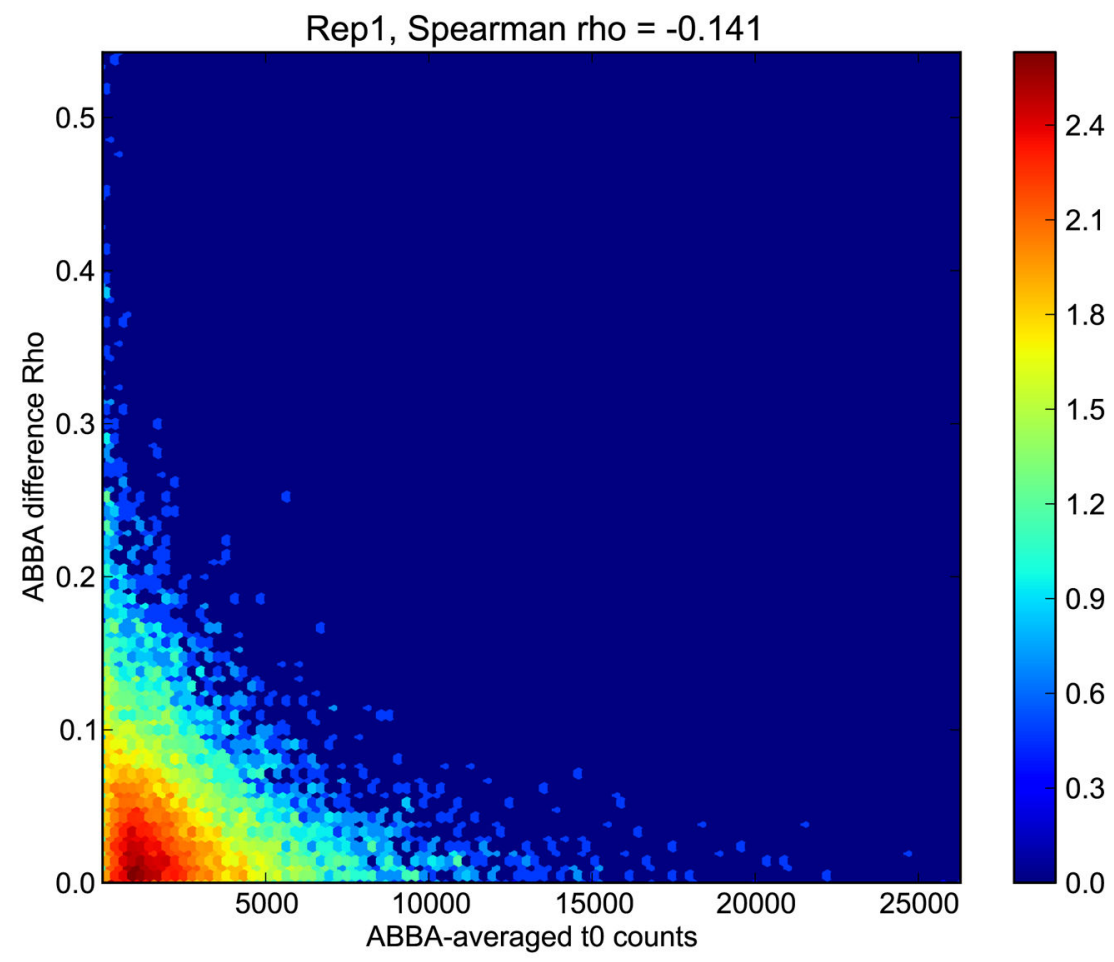

Figure 6.

Graphical output of the script double_shRNA_phenotypes.py (screenshot). A twodimensional histogram (implemented as hexagonally binned heatmap) of AB-BA differences in phenotype (rho) as a function of the average count numbers of the $\mathrm{AB}$ and $\mathrm{BA}$ double shRNAs in the t0 population. 


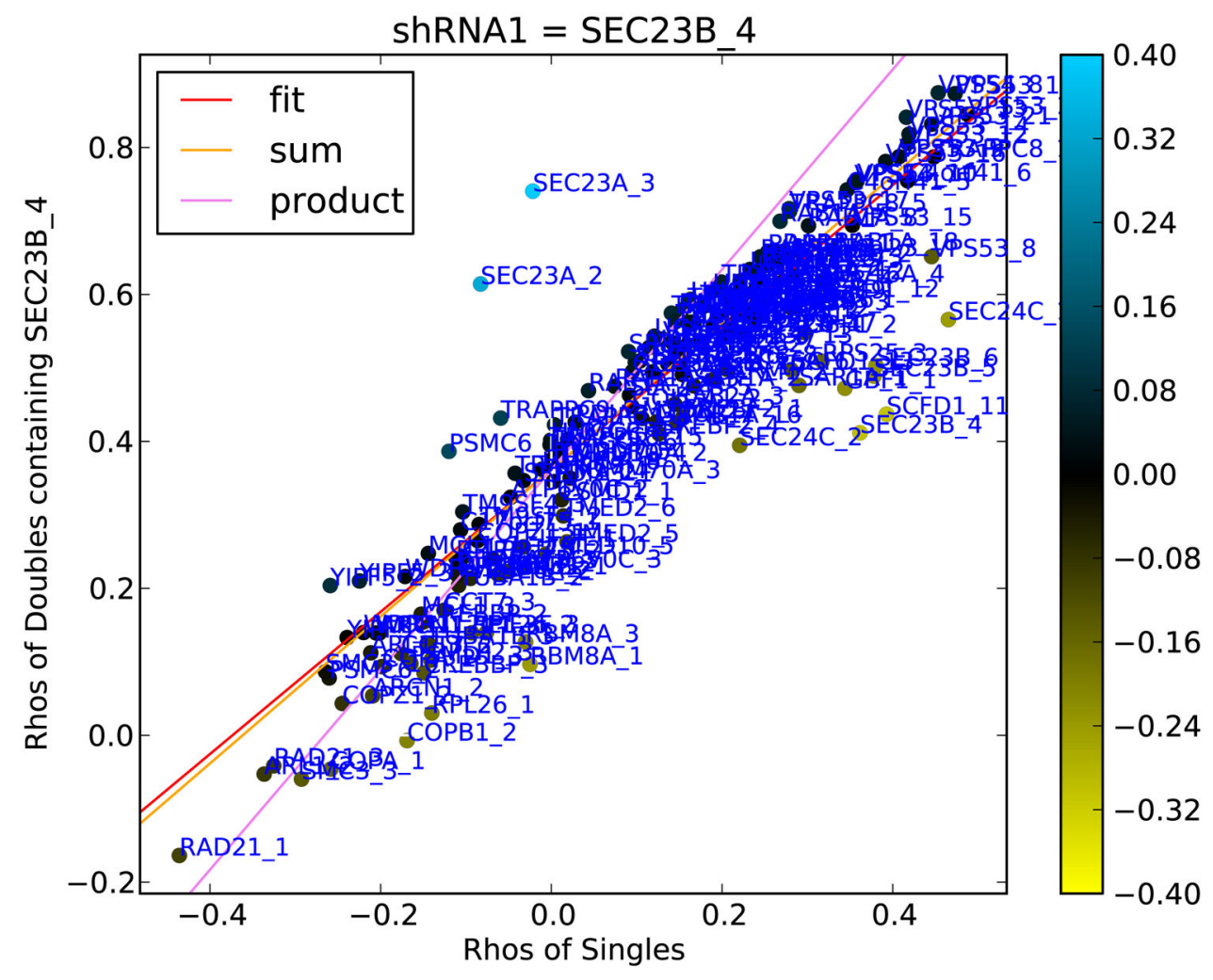

Figure 7.

Graphical output of the script calculate_GIs.py (screenshot). This graph was generated interactively using the ab_rho_sd_yellow_blue() command after running the script. Each dot represents an shRNA. X axis: average phenotype (rho) of double-shRNAs containing this shRNA and one of 12 negative control shRNAs. Y axis: phenotype (rho) of the doubleshRNA containing the same shRNA in combination with shRNA SEC23B_4. Values for shRNAs with SEC23B_4 in the first and second position of the shRNA were averaged. Additionally, expected double-shRNA phenotypes according to three different definitions are indicated by solid lines: red, linear fit orange, sum definition; pink, product definition. These definitions are detailed in ref. 9. 
Genetic interactions based on ricin resistance Synergistic $-0.2 \square 0.2$ Buffering

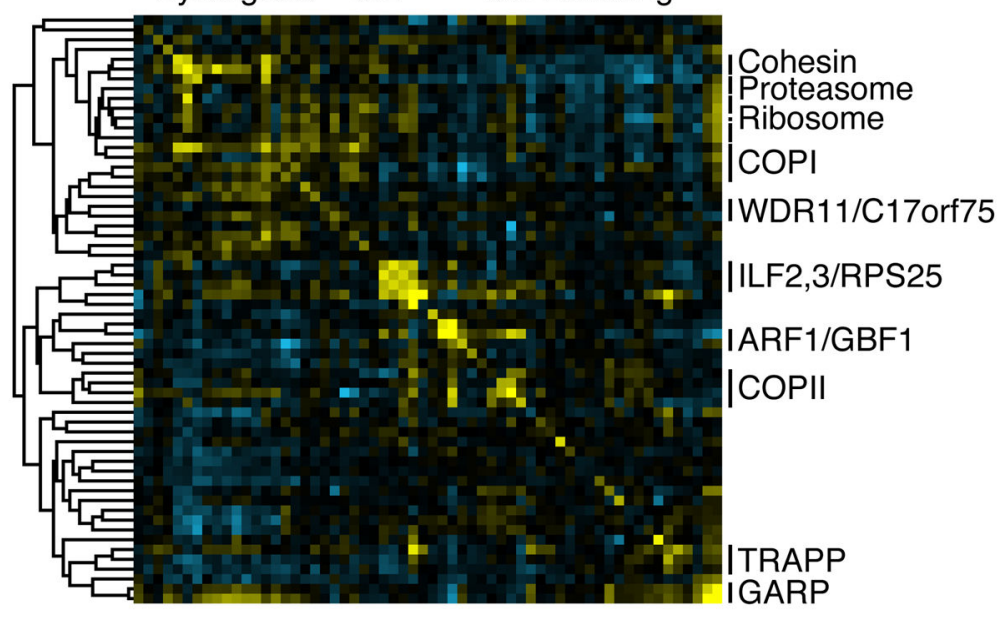

Figure 8.

High-density genetic interaction map created by hierarchical clustering of the genetic interaction matrix in Cluster 3.0 (ref. ${ }^{28}$ ), and displayed in Java Tree View ${ }^{29}$. Genes encoding proteins that interact physically (e.g. COPI complex subunits) or functionally (e.g. ARF1 and its nucleotide exchange factor GBF1) form clusters and typically show buffering genetic interactions with each other. 


\section{Oligonucleotide sequences}

\section{Table 1}

Illumina index sequences are shown in red.

\begin{tabular}{|c|c|c|}
\hline Name & Purpose & Oligonucleotide sequence ( $5^{\prime}$ to $\left.3^{\prime}\right)$ \\
\hline oMK312 & $\begin{array}{l}\text { Amplification of primary screen samples, forward } \\
\text { primer, Index } 1\end{array}$ & $\begin{array}{l}\text { aatgatacggcgaccaccgaGATCGG } \\
\text { AAGAGCACACGTCTGAACTCCAGTCA } \\
\text { CATCACGCTCTAGATGACTGACCCCTTG }\end{array}$ \\
\hline oMK313 & $\begin{array}{l}\text { Amplification of primary screen samples, forward } \\
\text { primer, Index } 2\end{array}$ & CCGATGTCTCTAGATGACTGACCCCTTG \\
\hline oMK314 & $\begin{array}{l}\text { Amplification of primary screen samples, forward } \\
\text { primer, Index } 9\end{array}$ & $\begin{array}{l}\text { aatgatacggcgaccaccgaGATCGG } \\
\text { AAGAGCACACGTCTGAACTCCAGTCA } \\
\text { CGATCAGCTCTAGATGACTGACCCCTTG }\end{array}$ \\
\hline oMK315 & $\begin{array}{l}\text { Amplification of primary screen samples, forward } \\
\text { primer, Index } 4\end{array}$ & $\begin{array}{l}\text { aatgatacggcgaccaccgaGATCGG } \\
\text { AAGAGCACACGTCTGAACTCCAGTCA } \\
\text { CTGACCACTCTAGATGACTGACCCCTTG }\end{array}$ \\
\hline oMK316 & $\begin{array}{l}\text { Amplification of primary screen samples, forward } \\
\text { primer, Index } 5\end{array}$ & $\begin{array}{l}\text { aatgatacggcgaccaccgaGATCGG } \\
\text { AAGAGCACACGTCTGAACTCCAGTCA } \\
\text { CACAGTGCTCTAGATGACTGACCCCTTG }\end{array}$ \\
\hline oMK317 & $\begin{array}{l}\text { Amplification of primary screen samples, forward } \\
\text { primer, Index } 6\end{array}$ & $\begin{array}{l}\text { aatgatacggcgaccaccgaGATCGG } \\
\text { AAGAGCACACGTCTGAACTCCAGTCA } \\
\text { CGCCAATCTCTAGATGACTGACCCCTTG }\end{array}$ \\
\hline oMK318 & $\begin{array}{l}\text { Amplification of primary screen samples, forward } \\
\text { primer, Index } 7\end{array}$ & $\begin{array}{l}\text { aatgatacggcgaccaccgaGATCGG } \\
\text { AAGAGCACACGTCTGAACTCCAGTCA } \\
\text { CCAGATCCTCTAGATGACTGACCCCTTG }\end{array}$ \\
\hline oMK319 & $\begin{array}{l}\text { Amplification of primary screen samples, forward } \\
\text { primer, Index } 13\end{array}$ & $\begin{array}{l}\text { aatgatacggcgaccaccgaGATCGG } \\
\text { AAGAGCACACGTCTGAACTCCAGTCA } \\
\text { CAGTCAACTCTAGATGACTGACCCCTTG }\end{array}$ \\
\hline oMCB800 & $\begin{array}{l}\text { Amplification of primary screen samples, reverse } \\
\text { primer }\end{array}$ & $\begin{array}{l}\text { CAAGCAGAAGACGGCATACGATGGAC } \\
\text { GAGCTGTACAAGTAA }\end{array}$ \\
\hline oMK132 & Deep sequencing primer for primary screen samples & ACCCCTTGAATTCCGAGGCAGTAGGCA \\
\hline $5^{\prime} \mathrm{pSico}-\mathrm{Eco}$ & Verification of inserts cloned into pMK1200 & CCACAACGAGGACTACACCA \\
\hline
\end{tabular}




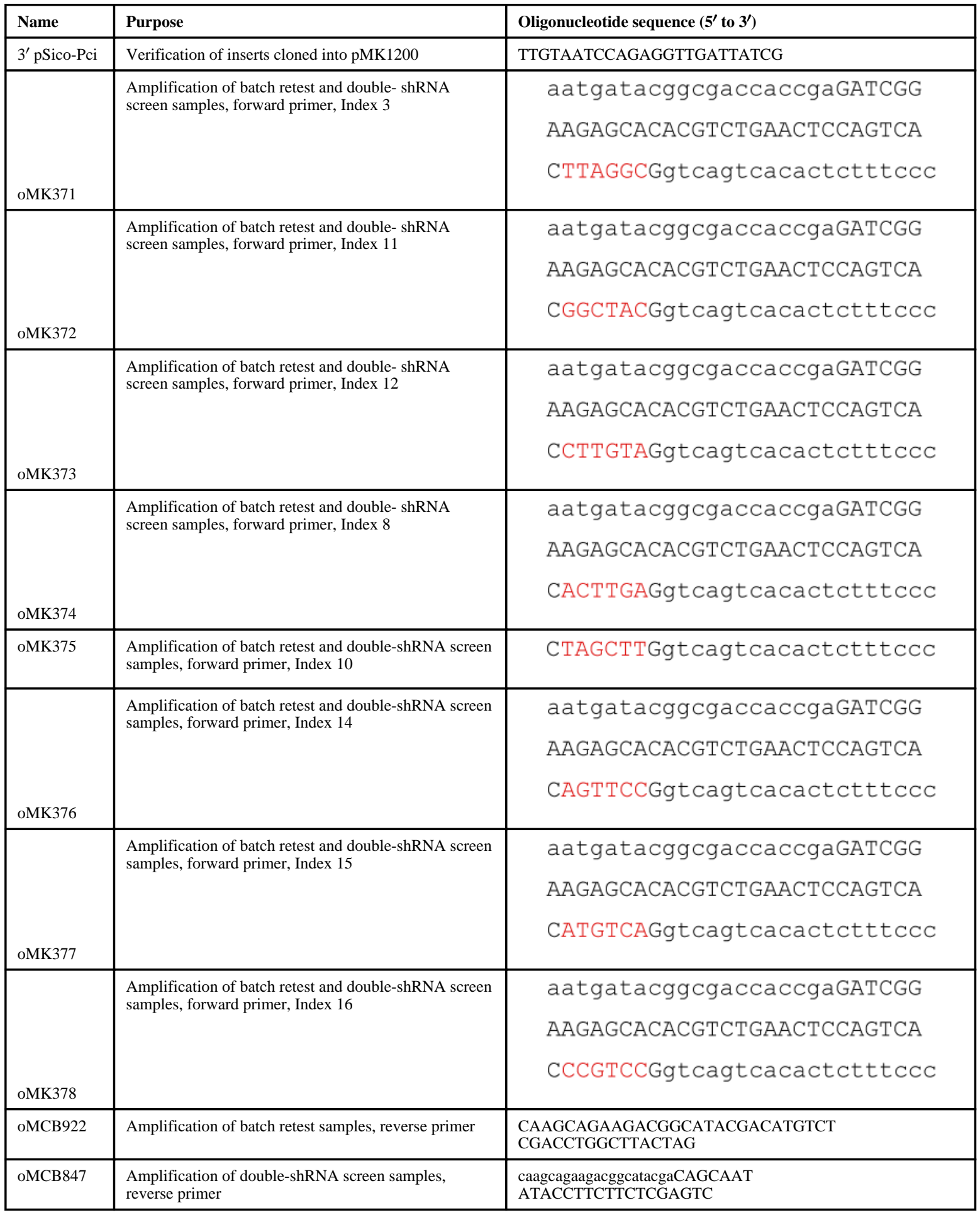




\section{Table 2}

Troubleshooting table.

\begin{tabular}{|c|c|c|c|}
\hline Step & Problem & Possible Reason & Possible Solution \\
\hline \multirow[t]{6}{*}{7} & \multirow[t]{6}{*}{ Low rate of infected cells } & \multirow[t]{4}{*}{ Problems with virus preparation } & Use fresh, low-passage 293T cells in Step 1. \\
\hline & & & Use fresh plasmid DNA in Step 2. \\
\hline & & & Use a new vial of Mirus LT1 in Step 2. \\
\hline & & & $\begin{array}{l}\text { Check for mCherry fluorescence in packaging cell } \\
\text { line in Step } 3 \text {. }\end{array}$ \\
\hline & & \multirow[t]{2}{*}{ Target cells are difficult to infect } & $\begin{array}{l}\text { Check infection of a control cell line (such as Hela } \\
\text { cells) }\end{array}$ \\
\hline & & & $\begin{array}{l}\text { Concentrate virus using spin-filtration or } \\
\text { ultracentrifugation after Step } 3 \text {. }\end{array}$ \\
\hline \multirow[t]{4}{*}{33} & \multirow[t]{3}{*}{$\begin{array}{l}\text { No or very faint band for expected } \\
\text { PCR product visible on ethidium } \\
\text { bromide-stained gel. }\end{array}$} & More PCR cycles are needed & $\begin{array}{l}\text { Increase number of PCR cycles in Step 28. However, } \\
\text { the number of PCR cycles should only be as high as } \\
\text { necessary to see a clear product band. Excessive } \\
\text { numbers of PCR cycles can introduce amplification } \\
\text { bias. }\end{array}$ \\
\hline & & $\begin{array}{l}\text { Too much genomic DNA was } \\
\text { used as template }\end{array}$ & $\begin{array}{l}\text { Reduce genomic DNA concentration to maximally } 1.5 \\
\mu \mathrm{g} \text { per } 100 \mu \mathrm{l} \text { in Step } 27 \text {. }\end{array}$ \\
\hline & & $\begin{array}{l}\text { General problems with PCR } \\
\text { reagents or thermocycler }\end{array}$ & $\begin{array}{l}\text { Run a positive control reaction in which the shRNA } \\
\text { library plasmid is used as a template. Use fresh } \\
\text { reagents in Step } 27 .\end{array}$ \\
\hline & Unexpected bands are observed & $\begin{array}{l}\text { Primer dimers and other template- } \\
\text { independent products. }\end{array}$ & $\begin{array}{l}\text { Perform and load a negative control reaction lacking } \\
\text { template on the gel next to the sample of interest. Run } \\
\text { the gel long enough to separate unwanted bands from } \\
\text { the band of interest. Cut out only the desired band in } \\
\text { Step } 33 \text { (see Fig. 3b) }\end{array}$ \\
\hline \multirow[t]{4}{*}{46} & $\begin{array}{l}\text { The distribution of negative control } \\
\text { shRNA phenotypes is dramatically } \\
\text { wider than in the example shown in } \\
\text { Fig. } 4 .\end{array}$ & $\begin{array}{l}\text { At least one of the cell populations } \\
\text { underwent a bottleneck during the } \\
\text { screen or sample preparation }\end{array}$ & $\begin{array}{l}\text { Repeat or redesign the screening protocol (Step 8) to } \\
\text { avoid population bottlenecks. }\end{array}$ \\
\hline & \multirow[t]{3}{*}{$\begin{array}{l}\text { shRNA phenotypes show a bimodal } \\
\text { distribution or have a very broad } \\
\text { "shoulder" on one side of the } \\
\text { distribution. }\end{array}$} & \multirow[t]{2}{*}{$\begin{array}{l}\text { In at least one of the cell } \\
\text { populations, bias was introduced } \\
\text { during the sample preparation. }\end{array}$} & $\begin{array}{l}\text { Make sure to use the same number of PCR cycles in } \\
\text { Step } 28 \text { for all samples that will be compared } \\
\text { computationally. }\end{array}$ \\
\hline & & & $\begin{array}{l}\text { If the primary shRNA library contains shRNAs of } \\
\text { different lengths (e.g. } 21 \text { mers and } 22 \text { mers), it is } \\
\text { important to avoid introducing size bias when cutting } \\
\text { bands from the polyacrylamide gels (Step 33). } \\
\text { Therefore, the band should be cut out very } \\
\text { generously, leaving a margin of unstained gel around } \\
\text { the band. }\end{array}$ \\
\hline & & $\begin{array}{l}\text { At least one of the cell populations } \\
\text { was contaminated with a different } \\
\text { sample. }\end{array}$ & $\begin{array}{l}\text { Use separate equipment for pre-PCR steps (Steps 11- } \\
\text { 28) and post-PCR steps (Steps 29-38). In particular, } \\
\text { use separate vacuum manifolds. }\end{array}$ \\
\hline 60 & $\begin{array}{l}\text { Same problems as for the primary } \\
\text { screen (Troubleshooting for Steps } 7 \text {, } \\
33 \text { above). }\end{array}$ & $\begin{array}{l}\text { See possible reasons for } \\
\text { corresponding problem in primary } \\
\text { screen (Troubleshooting for Steps } \\
7,33 \text { above). }\end{array}$ & $\begin{array}{l}\text { See possible reasons for corresponding solution in } \\
\text { primary screen (Troubleshooting for Steps } 7,33 \\
\text { above). }\end{array}$ \\
\hline 77 & $\begin{array}{l}\text { Same problems as for the primary } \\
\text { screen (Troubleshooting for Steps } 7 \text {, } \\
33 \text { above). }\end{array}$ & $\begin{array}{l}\text { See possible reasons for } \\
\text { corresponding problem in primary } \\
\text { screen (Troubleshooting for Steps } \\
7,33 \text { above). }\end{array}$ & $\begin{array}{l}\text { See possible reasons for corresponding solution in } \\
\text { primary screen (Troubleshooting for Steps } 7,33 \\
\text { above). }\end{array}$ \\
\hline 86 & $\begin{array}{l}\text { Large differences between } \mathrm{AB} \text { and } \\
\mathrm{BA} \text { phenotypes for a given } \\
\text { combination of shRNAs A, B. }\end{array}$ & $\begin{array}{l}\text { See possible reasons for } \\
\text { corresponding problem in primary } \\
\text { screen (Troubleshooting for Steps } \\
46 \text { above). }\end{array}$ & $\begin{array}{l}\text { See possible reasons for corresponding solution in } \\
\text { primary screen (Troubleshooting for Steps } 46 \text { above). }\end{array}$ \\
\hline
\end{tabular}

